\title{
Universally-convergent Squared-operator Iteration Methods for Solitary Waves in General Nonlinear Wave Equations
}

\author{
Jianke Yang ${ }^{\dagger, \dagger}$ and Taras I. Lakoba ${ }^{\dagger}$ \\ $\dagger$ : Department of Mathematics and Statistics, University of Vermont, Burlington, VT 05401 \\ $\dagger \dagger$ : Zhou Pei-Yuan Center for Applied Mathematics, Tsinghua University, Beijing, China
}

Key words: nonlinear evolution equations, solitary waves, iteration methods, convergence.

Summary:

Three new iteration methods, namely the squared-operator method, the modified squaredoperator method, and the power-conserving squared-operator method, for solitary waves in general scalar and vector nonlinear wave equations are proposed. These methods are based on iterating new differential equations whose linearization operators are squares of those for the original equations, together with acceleration techniques. The first two methods keep the propagation constants fixed, while the third method keeps the powers (or other arbitrary functionals) of the solution fixed. It is proved that all these methods are guaranteed to converge to any solitary wave (either ground state or not) as long as the initial condition is sufficiently close to the corresponding exact solution, and the time step in the iteration schemes is below a certain threshold value. Furthermore, these schemes are fast-converging, highly accurate, and easy to implement. If the solitary wave exists only at isolated propagation constant values, the corresponding squared-operator methods are developed as well. These methods are applied to various solitary wave problems of physical interest, such as higher-gap vortex solitons in the two-dimensional nonlinear Schrödinger equations with periodic potentials, and isolated solitons in Ginzburg-Landau equations, and some new types of solitary wave solutions are obtained. It is also demonstrated that the modified squared-operator method delivers the best performance among the methods proposed in this article.

\section{Introduction}

Solitary waves play an important role in nonlinear wave equations. While such waves in some wave equations can be obtained analytically (such as in integrable equations), they defy analytical expressions in most other cases. Thus numerical computations of solitary waves is an important issue. In the past, a number of numerical methods have been de- 
veloped for solitary waves. One of them is Newton's iteration method. This method can converge very fast. However, it often employs the finite-difference discretization, which has a low accuracy (compared to spectral or pseudospectral methods). In addition, in higher dimensions, time-efficient programming of this method becomes more cumbersome. Recent studies also showed that this method can suffer erratic failures due to small denominators [1]. Another method is the shooting method (see [2] for instance). This method works for all one-dimensional problems and higher-dimensional problems which can be reduced to the one-dimensional problem (by symmetry reduction, for instance). It is efficient and highly accurate. However, it fails completely for higher-dimensional problems which are not reducible to the one-dimensional problem. A third method is the nonlinear Rayleigh-Ritz iteration method (also called the self-consistency method) [3, 4], where one treats the nonlinear eigenvalue problem as a linear one with a solution-dependent potential. The solitary wave is obtained by repeatedly solving the linear eigenvalue problem and normalizing the solution. This method can become cumbersome as well in high dimensions when the linear eigenvalue problem becomes harder to solve. Two more methods are the Petviashvili method $[5,6,7,8]$ and the imaginary-time evolution method $[9,10,11,12,13]$. The convergence properties of the former method were studied in [6] for a class of equations with power nonlinearity, while those of the latter method were obtained in [13] for a much larger class of equations with arbitrary nonlinearity. Interestingly, it was shown that the convergence of the latter method is directly linked to the linear stability of the solitary wave if the solitary wave is sign-definite [13]. Both methods converge fast, are highly accurate, are insensitive to the number of dimensions, and their performances are comparable [13]. However, both methods diverge for many solitary waves (especially ones which cross zeros, i.e., excited states) $[6,13]$. In recent years, some interesting yet challenging solitary wave problems arise in physical applications. Two notable examples are nonlinear light propagation in multi-dimensional periodic and quasi-periodic media, and Bose-Einstein condensates loaded in multi-dimensional harmonic magnetic traps and periodic optical lattices. These problems are not reducible to one-dimensional problems, so the shooting method can not be used. In addition, solitary waves in these problems often cross zeros (as is always the case in higher bandgaps), thus the Petviashvili method and the imaginary-time evolution method do not converge. Furthermore, the numerical stability analysis of such solutions require the solutions themselves to have high accuracy, which is often hard to achieve by the Newton's method or the self-consistency method. Thus new numerical schemes are called upon. These schemes should be time-efficient, highly accurate, insensitive to the number of dimensions, and capable of computing all types of solitary waves in any scalar or vector nonlinear wave equations. Equally importantly, these schemes should be easy to implement. None of the previous methods meets all these requirements. 
In this paper, we propose several classes of iteration methods for solitary waves which do meet all the requirements described above. These methods are based on two key ideas. The first key idea is to iterate a modified equation whose linearization operator is a square of that for the original equation. This idea (in a different form) was first presented in [9]. Specifically, those authors proposed to obtain excited-state solitary waves as stationary points of a certain functional that is different from the usual Lagrangian. In the present study, we will show that this procedure is equivalent to using the aforementioned squared operator in the iteration equation. We further show that this operator structure guarantees that the proposed methods converge for all types of solitary waves in any nonlinear wave equations. These iteration methods are compatible with the pseudo-spectral method for the computation of spatial derivatives, thus they are highly accurate (with errors that decrease exponentially with the decrease of the spatial grid size), and can handle multi-dimensional problems with little change in the programming. The second key idea is to introduce an acceleration operator to the scheme $[9,13]$. The acceleration operator speeds up the convergence of these iteration methods drastically, hence making them highly time-efficient. Based on these two key ideas, we propose two powerful new iteration methods which we call the squared-operator method and the power(or arbitrary quantity)-conserving squaredoperator method. The former method specifies the propagation constant of the solitary wave, while the latter method specifies the power or other arbitrary functional of the solution. Both methods are shown to converge to any solitary wave if the time-step parameter in the methods is below a certain threshold value, and the initial condition is sufficiently close to the exact solution. Beyond these two ideas, we also employ an eigenmode-elimination technique [14] which speeds up the convergence of iterations even further. The resulting numerical method will be called the modified squared-operator method in the text. All these schemes pertain to solitary waves which exist at continuous propagation constants. In certain nonlinear wave problems (especially of dissipative nature), however, solitary waves exist at isolated propagation constants. By extending the above ideas, we construct the squared-operator method and the modified squared-operator method for isolated solitary waves as well. By applying these new schemes to various challenging solitary wave problems, we demonstrate that the modified squared-operator method gives the best performance, followed by the squared-operator method, then followed by the power-conserving squared-operator method. In the end, we construct a whole family of iteration schemes which share similar convergence properties and contain the presented schemes as special cases.

This paper is structured as follows. In Section 2, we present the squared-operator method and show that it converges for all types of solitary waves in arbitrary vector equations. We 
also demonstrate it on the nonlinear Schrödinger equation where we carry out the explicit convergence analysis and determine optimal parameters in the acceleration operator. In Section 3, we present the modified squared-operator method and show that this method not only converges for all types of solitary waves in arbitrary equations, but also converge faster than the squared-operator method. In Section 4, we present power-conserving versions of the squared-operator method and prove their universal convergence properties. In Section 5, we derive the squared-operator method and the modified squared-operator method for isolated solitary waves. In Section 6, we demonstrate all these methods on various challenging examples, including vortex-array solitons in higher bandgaps and solitary waves in coherent three-wave interactions, and show that the modified squared-operator method delivers the best performance. In the Appendix, we present whole families of squared-operator-like methods, and show that the methods described in the main text are the leading members in these families in terms of convergence speeds.

\section{The squared-operator method for solitary waves in general nonlinear wave equations}

Consider solitary waves in a general real-valued coupled nonlinear wave system in arbitrary spatial dimensions, which can be written in the following form

$$
\mathbf{L}_{0} \mathbf{u}(\mathbf{x})=0
$$

Here $\mathbf{x}$ is a vector spatial variable, $\mathbf{u}(\mathbf{x})$ is a real-valued vector solitary wave solution admitted by Eq. (1), and $\mathbf{u} \rightarrow 0$ as $|\mathbf{x}| \rightarrow \infty$. Note that for complex-valued solitary waves, the equation can be rewritten in the above form with $\mathbf{u}$ containing the real and imaginary parts of the complex solution. Let $\mathbf{L}_{1}$ denote the linearized operator of Eq. (1) around the solution $\mathbf{u}$, i.e.,

$$
\mathbf{L}_{0}(\mathbf{u}+\tilde{\mathbf{u}})=\mathbf{L}_{1} \tilde{\mathbf{u}}+O\left(\tilde{\mathbf{u}}^{2}\right), \quad \tilde{\mathbf{u}} \ll 1,
$$

$(\cdot)^{\dagger}$ denote the Hermitian of the underlying quantity, and $\mathbf{M}$ be a real-valued positivedefinite Hermitian operator which we call the acceleration operator. The idea of our method is to numerically integrate the time-dependent equation

$$
\mathbf{u}_{t}=-\mathbf{M}^{-1} \mathbf{L}_{1}^{\dagger} \mathbf{M}^{-1} \mathbf{L}_{0} \mathbf{u}
$$

rather than $\mathbf{u}_{t}= \pm \mathbf{M}^{-1} \mathbf{L}_{0} \mathbf{u}$. The reason is to guarantee the convergence of numerical integrations, as we will demonstrate below. The operator $\mathbf{M}$ is introduced to speed up the 
convergence, in the same spirit as the pre-conditioning technique in solving systems of linear algebraic equations. Using the simplest time-stepping method for Eq. (3) - the forward Euler method, the iteration method we propose for computing solitary waves $\mathbf{u}$ is

$$
\mathbf{u}_{n+1}=\mathbf{u}_{n}-\left[\mathbf{M}^{-1} \mathbf{L}_{1}^{\dagger} \mathbf{M}^{-1} \mathbf{L}_{0} \mathbf{u}\right]_{\mathbf{u}=\mathbf{u}_{n}} \Delta t
$$

It will be shown below that this method is universally convergent as long as the time step $\Delta t$ is below a certain threshold, and this universal convergence stems from the fact that the iteration operator for the error function is $-\mathbf{M}^{-1} \mathbf{L}_{1}^{\dagger} \mathbf{M}^{-1} \mathbf{L}_{1}$, or "square" of the operator $\mathbf{M}^{-1} \mathbf{L}_{1}$. Thus we call scheme (4) the squared-operator method (SOM). If $\mathbf{M}$ is taken as the identity operator (no acceleration), then SOM (4) reduces to

$$
\mathbf{u}_{n+1}=\mathbf{u}_{n}-\left[\mathbf{L}_{1}^{\dagger} \mathbf{L}_{0} \mathbf{u}\right]_{\mathbf{u}=\mathbf{u}_{n}} \Delta t
$$

which has a simpler appearance but converges very slowly.

It should be noted that even though more complicated time-stepping methods (such as Runge-Kutta methods) can also be used to integrate Eq. (3), they are actually less efficient than the forward Euler method (4), because the extra computations in them outweighs the benefits they may have. Implicit methods are even less attractive. The reason is that due to the acceleration operator $\mathbf{M}$, the time steps $\Delta t$ allowed by explicit methods such as (4) for numerical stability (or convergence) are not small, thus the need for implicit methods vanishes.

Scheme (4) is actually one of the many SOM's one can construct using the same squaredoperator idea. Indeed, in the Appendix, we will present a whole family of SOM's, of which (4) is a particular member. We will show there that scheme (4) is the leading member in that family in terms of convergence speeds.

Let us now remark on the relation of these methods to the functional minimization method for Hamiltonian equations proposed in [9], which has the following form:

$$
\mathbf{u}_{t}=-\frac{\delta}{\delta \mathbf{u}} \int\left\|\mathbf{L}_{0} \mathbf{u}\right\|^{2} d \mathbf{x}
$$

Here $\delta / \delta \mathbf{u}$ represents the functional (Frechet) derivative, and $\|\ldots\|$ denotes the $L_{2}$-norm. Upon taking this functional derivative and noticing that for Hamiltonian equations, $\mathbf{L}_{1}^{\dagger}=$ $\mathbf{L}_{1}$, one recovers Eq. (3) with $\mathbf{M}=1$. The accelerated equation (3) follows similarly from the functional equation

$$
\hat{\mathbf{u}}_{t}=-\frac{\delta}{\delta \hat{\mathbf{u}}} \int\left\|\mathbf{M}^{-1 / 2} \mathbf{L}_{0} \mathbf{M}^{-1 / 2} \hat{\mathbf{u}}\right\|^{2} d \mathbf{x}
$$


where $\hat{\mathbf{u}}=\mathbf{M}^{1 / 2} \mathbf{u}$.

To formulate the convergence theorem for the SOM (4), we introduce the following assumption.

Assumption 1 Let $\boldsymbol{u}\left(\mathbf{x} ; c_{1}, c_{2}, \ldots, c_{s}\right)$ be the general solution of Eq. (1), where $\left(c_{1}, c_{2}, \ldots, c_{s}\right)$ are free real parameters. Then we assume that the only eigenfunctions in the kernel of $\mathbf{L}_{\mathbf{1}}$ are the $s$ invariance modes $\boldsymbol{u}_{c_{j}}, 1 \leq j \leq s$, where $\boldsymbol{u}_{c_{j}}=\partial \boldsymbol{u} / \partial c_{j}$.

Remark 2.1 One example of these invariance modes is the translational-invariance mode. Suppose Eq. (1) is translationally invariant along the direction $x_{1}$, i.e., $\mathbf{u}\left(x_{1}+c_{1}, x_{2}, \ldots, x_{N}\right)$ is a solution for any value of $c_{1}$. Then the translational-invariance mode $\mathbf{u}_{c_{1}}=\mathbf{u}_{x_{1}}$ is in the kernel of $\mathbf{L}_{\mathbf{1}}$. Another example of the invariance modes pertains to arbitrary phases of complex-valued solutions and can be found, e.g., in Example 6.1 below.

Remark 2.2 Assumption 1 holds in the generic case. However, in certain non-generic cases, it does break down (see Fig. 7 of [15] for an example).

Under Assumption 1, we have the following theorem.

Theorem 1 Let Assumption 1 be valid, and define

$$
\Delta t_{\max } \equiv-\frac{2}{\Lambda_{\min }}
$$

where $\Lambda_{\min }$ is the minimum eigenvalue of operator

$$
\mathcal{L} \equiv-\boldsymbol{M}^{-1} \boldsymbol{L}_{1}^{\dagger} \boldsymbol{M}^{-1} \boldsymbol{L}_{1}
$$

Then SOM (4) is guaranteed to converge to the solitary wave $\boldsymbol{u}(\mathbf{x})$ of $E q$. (1) if $\Delta t<\Delta t_{\text {max }}$ and the initial condition is close to $\boldsymbol{u}(\mathbf{x})$. If $\Delta t>\Delta t_{\text {max }}$, then $S O M$ (4) diverges from $\boldsymbol{u}(\mathbf{x})$.

Proof. We use the linearization technique to analyze SOM (4) and prove the theorem. Let

$$
\mathbf{u}_{n}=\mathbf{u}+\tilde{\mathbf{u}}_{n}, \quad \tilde{\mathbf{u}}_{n}(\mathbf{x}) \ll 1,
$$

where $\tilde{\mathbf{u}}_{n}(\mathbf{x})$ is the error. When Eq. (10) is substituted into SOM (4) and only terms of $O\left(\tilde{\mathbf{u}}_{n}\right)$ are retained, we find that the error satisfies the following linear equation:

$$
\tilde{\mathbf{u}}_{n+1}=(1+\Delta t \mathcal{L}) \tilde{\mathbf{u}}_{n}
$$


where $\mathcal{L}$ is defined in Eq. (9). Note that since $\mathcal{L}=\mathbf{M}^{-1 / 2} \mathcal{L}_{h} \mathbf{M}^{1 / 2}$, where

$$
\mathcal{L}_{h}=-\left(\mathbf{M}^{-1 / 2} \mathbf{L}_{1} \mathbf{M}^{-1 / 2}\right)^{\dagger}\left(\mathbf{M}^{-1 / 2} \mathbf{L}_{1} \mathbf{M}^{-1 / 2}\right)
$$

is Hermitian and semi-negative-definite, thus all eigenvalues of $\mathcal{L}$ are real and non-positive. In addition, all eigenfunctions of $\mathcal{L}$ form a complete set in the square-integrable functional space, hence $\tilde{\mathbf{u}}_{n}$ can be expanded over $\mathcal{L}$ 's eigenfunctions. Consequently, if $\Delta t>\Delta t_{\text {min }}$, the eigenmode with eigenvalue $\Lambda_{\min }$ in the error will grow exponentially due to $1+\Lambda_{\min } \Delta t<-1$, i.e., SOM (4) diverges. On the other hand, if $\Delta t<\Delta t_{\text {min }}$, then no eigenmode in the error can grow. In fact, all eigenmodes decay with iterations except those in the kernel of $\mathcal{L}$. But according to Assumption 1, eigenfunctions in the kernel of $\mathcal{L}$ are all invariance modes $\mathbf{u}_{c_{j}}$ which only lead to another solution with slightly shifted constants $c_{j}$ and do not affect the convergence of iterations. Thus Theorem 1 is proved.

Remark 2.3 The role of the acceleration operator $\mathbf{M}$ is to make $\Lambda_{\min }$ of $\mathcal{L}$ bounded (without $\mathbf{M}, \Lambda_{\min }=-\infty$ in general); see, e.g., Example 2.1 below and [13]. As shown after Remark 2.4 below, this leads to faster convergence of SOM (4).

Remark 2.4 In computer implementations of SOM (4), spatial discretization is used. In such a case, if we require the discretization $\mathbf{M}^{(D)}$ of the positive-definite Hermitian operator $\mathbf{M}$ to remain positive-definite and Hermitian (which is needed to show the non-positiveness of eigenvalues of the discretized operator $\mathcal{L}^{(D)}$ ), then Theorem 1 and its proof can be readily extended to the spatially-discretized SOM scheme (4), except that $\Lambda_{\min }$ in Eq. (8) becomes the smallest eigenvalue of the discrete operator $\mathcal{L}^{(D)}$ now. Unlike discretizations of some other schemes such as the Petviashvili method [6] and the accelerated imaginary-time evolution method [13], discretizations of SOM (4) always converge regardless of whether the discretized solitary wave is site-centered (on-site) or inter-site-centered (off-site). Note that under discretization, the kernel of $\mathcal{L}^{(D)}$ may become smaller than that of $\mathcal{L}$ due to the breakdown of translational invariances, but this does not affect the extension of Theorem 1 and its proof to the discretized SOM scheme at all.

In the application of SOM (4), a practical question is how to choose $\Delta t$ within the upper bound of $\Delta t_{\max }$ so that convergence is the fastest. To answer this question, let us define the convergence factor $R$ of SOM (4) as

$$
R \equiv \max _{\Lambda}\{|1+\Lambda \Delta t|\}
$$

where $\Lambda$ is any non-zero eigenvalue of $\mathcal{L}$. Then the error $\tilde{\mathbf{u}}_{n}$ of the iterated solution decays as $R^{n}$, where $n$ is the number of iterations. Smaller $R$ gives faster convergence. Clearly,

$$
R=\max \left\{\left|1+\Lambda_{\min } \Delta t\right|,\left|1+\Lambda_{\max } \Delta t\right|\right\},
$$


where $\Lambda_{\max }$ is the largest non-zero eigenvalue of operator $\mathcal{L}$. From this equation, we see that the smallest $R$ (fastest convergence) occurs at the time step

$$
\Delta t=\Delta t_{*} \equiv-\frac{2}{\Lambda_{\min }+\Lambda_{\max }}
$$

for which the corresponding convergence factor is

$$
R_{*}=\frac{\Lambda_{\min }-\Lambda_{\max }}{\Lambda_{\min }+\Lambda_{\max }}
$$

Another practical question which arises in the implementation of SOM (4) is the choice of the acceleration operator $\mathbf{M}$. This operator should be chosen such that $\mathbf{M}$ is easily invertible. In addition, it should be chosen such that the convergence is maximized or nearly maximized. A simple but often effective choice is to take $\mathbf{M}$ in the form of the linear part of the original equation (1), to which one adds a constant to make it positive definite.

To demonstrate the effect of $\mathbf{M}$ on convergence speeds, below we consider the familiar nonlinear Schrödinger (NLS) equation for which the convergence analysis of SOM can be done explicitly.

Example 2.1 Consider the NLS equation in one spatial dimension:

$$
u_{x x}+u^{3}=\mu u \text {. }
$$

Without loss of generality, we take $\mu=1$. For this $\mu$ value, Eq. (16) has a soliton solution

$$
u(x)=\sqrt{2} \operatorname{sech} x .
$$

We take the acceleration operator $M$ to be in the form of the linear part of Eq. (16), i.e.,

$$
M=c-\partial_{x x}, \quad c>0,
$$

which is easily invertible using the Fourier transform. Then the eigenvalue equation for operator $M^{-1} L_{1}$,

$$
M^{-1} L_{1} \psi=\lambda \psi,
$$

can be rewritten in the explicit form:

$$
\psi_{x x}-\frac{1+c \lambda}{1+\lambda} \psi+\frac{6}{1+\lambda} \operatorname{sech}^{2} x \psi=0 .
$$


The continuous spectrum of this equation can be obtained by requiring the eigenfunction to be oscillatory at $|x|=\infty$, and this continuous spectrum is found to be

$$
\begin{aligned}
& \lambda \in\left(-1,-\frac{1}{c}\right], \text { for } c>1 ; \\
& \lambda \in\left[-\frac{1}{c},-1\right), \text { for } c<1 .
\end{aligned}
$$

The discrete eigenvalues of (20) satisfy the equation $[16,17]$

$$
\sqrt{\frac{1+c \lambda}{1+\lambda}}=\frac{1}{2}\left[\sqrt{1+\frac{24}{1+\lambda}}-(2 j+1)\right], \quad j=0,1,2, \ldots,
$$

where the right hand side should be non-negative. These discrete eigenvalues are plotted in Fig. 1(a). Note that $\lambda=0$ is always a discrete eigenvalue with $j=1$. This zero eigenvalue is due to the translational invariance of the NLS soliton (which leads to $L_{1} u_{x}=0$ ). The eigenvalues $\lambda_{0}$ and $\lambda_{2}$ (for $j=0$ and 2) can be found to have the following expressions:

$$
\begin{gathered}
\lambda_{0}(c)=\frac{2(c+5)^{2}}{2 c^{2}+9 c+1+\sqrt{25 c^{2}+118 c+1}}-1, \\
\lambda_{2}(c)=\frac{2(c+5)^{2}}{2 c^{2}-3 c+85+5 \sqrt{25 c^{2}-26 c+145}}-1 .
\end{gathered}
$$

From the above spectrum of operator $M^{-1} L_{1}$, we can easily obtain the spectrum of the iteration operator $\mathcal{L}=-M^{-1} L_{1}^{\dagger} M^{-1} L_{1}$, whose eigenvalues $\Lambda$ are related to the eigenvalues $\lambda$ of $M^{-1} L_{1}$ as $\Lambda=-\lambda^{2}$, since in this case $L_{1}^{\dagger}=L_{1}$. Hence

$$
\Lambda_{\max }(c)=-\lambda_{2}^{2}(c), \quad \Lambda_{\min }(c)=\min \left\{-\lambda_{0}^{2}(c),-\frac{1}{c^{2}},-1\right\} .
$$

These eigenvalues are plotted in Fig. 1(b). Based on these eigenvalues, we can calculate the convergence factor $R_{*}(c)$ from formula (15). This $R_{*}(c)$ is shown in Fig. 1(c). It is seen that when $\lambda_{0}^{2}(c)=1$, i.e., $c=c_{\text {opt }}=6-\sqrt{13} \approx 2.4, R_{*}(c)$ reaches its minimum value $R_{\text {min }} \approx 0.80$. At $c=c_{\text {opt }}, R_{*}(c)$ is not differentiable, because $\Lambda_{\min }(c)$ equals $-\lambda_{0}^{2}(c)$ for $c<c_{o p t}$ and -1 (the edge of the continuous spectrum) for $c>c_{\text {opt }}$. For $c=c_{\text {opt }}$, the dependence of the convergence factor $R$ on $\Delta t$ is shown in Fig. 1(d). At $\Delta t_{*} \approx 1.80$ from formula (14), $R$ reaches its minimum value $R_{\text {min }}$ given above. Without acceleration $(M=1)$, this value of $R$ would be very close to 1 with discretizations (since $\Lambda_{\min }$ is large negative, see [13]), and be exactly equal to 1 without discretizations ( since $\left.\Lambda_{\min }=-\infty\right)$. From the above explicit analysis, we see that the choice of $M$ affects the convergence speed of SOM (4) significantly. 


\section{The modified squared-operator method for solitary waves in general nonlinear wave equations}

The above SOM (4), even with a sensible choice of the acceleration operator $\mathbf{M}$, can still be quite slow for certain problems (see the example in Fig. 4). In this section, we employ an additional technique which can speed up the convergence of SOM iterations even further. This technique is called eigenmode elimination, and was originally proposed in [14] for a non-squared-operator scheme. When this eigenmode-elimination technique is incorporated into SOM, the resulting method, which we call as the modified squared-operator method (MSOM), will converge faster than SOM (4).

The MSOM we propose is

$$
\mathbf{u}_{n+1}=\mathbf{u}_{n}-\left[\mathbf{M}^{-1} \mathbf{L}_{1}^{\dagger} \mathbf{M}^{-1} \mathbf{L}_{0} \mathbf{u}-\alpha_{n}\left\langle\mathbf{G}_{n}, \mathbf{L}_{1}^{\dagger} \mathbf{M}^{-1} \mathbf{L}_{0} \mathbf{u}\right\rangle \mathbf{G}_{n}\right]_{\mathbf{u}=\mathbf{u}_{n}} \Delta t
$$

where

$$
\alpha_{n}=\frac{1}{\left\langle\mathbf{M G}_{n}, \mathbf{G}_{n}\right\rangle}-\frac{1}{\left\langle\mathbf{L}_{1} \mathbf{G}_{n}, \mathbf{M}^{-1} \mathbf{L}_{1} \mathbf{G}_{n}\right\rangle \Delta t},
$$

$\mathbf{G}_{n}$ is a function the user can specify, and the inner product is the standard one in the square-integrable functional space:

$$
\left\langle\mathbf{F}_{1}, \mathbf{F}_{2}\right\rangle=\int_{-\infty}^{\infty} \mathbf{F}_{1}^{\dagger} \cdot \mathbf{F}_{2} d \mathbf{x}
$$

If $\left\langle\mathbf{F}_{1}, \mathbf{F}_{2}\right\rangle=0, \mathbf{F}_{1}$ and $\mathbf{F}_{2}$ are said to be orthogonal to each other. Two simple choices for $\mathbf{G}_{n}$ can be

$$
\mathbf{G}_{n}=\mathbf{u}_{n}
$$

and

$$
\mathbf{G}_{n}=\mathbf{e}_{n} \equiv \mathbf{u}_{n}-\mathbf{u}_{n-1}
$$

The motivation for MSOM (1) can be explained briefly as follows [14]. Consider SOM (4), and denote the slowest-decaying eigenmode of $\mathcal{L}$ in the error as $\mathbf{G}(\mathbf{x})$ with eigenvalue $\Lambda_{s}$. Note that according to Eqs. (12) and (13), $\Lambda_{s}=\Lambda_{\min }$ or $\Lambda_{\max }$. Our idea is to construct a modified linearized iteration operator for the error so that eigenmode $\mathbf{G}(\mathbf{x})$ decays quickly, while the decay rates of other eigenmodes of $\mathcal{L}$ remain the same. If so, then this modified iteration scheme would converge faster than the original SOM. For this purpose, consider the modified linearized iteration operator

$$
\mathcal{L}_{M} \Psi=\mathcal{L} \Psi-\alpha\langle\mathbf{M G}, \mathcal{L} \Psi\rangle \mathbf{G}
$$


where $\alpha$ is a constant. Since $\mathbf{G}(\mathbf{x})$ is an eigenfunction of $\mathcal{L}$, and recalling that eigenfunctions of this $\mathcal{L}$ are orthogonal to each other under the $\mathbf{M}$-weighted inner product, we readily see that this modified iteration operator and the original one have identical eigenfunctions. Their eigenvalues are identical too except the one for eigenmode $\mathbf{G}(\mathbf{x})$. The eigenvalue of this eigenmode changes from $\Lambda_{s}$ of $\mathcal{L}$ to $(1-\alpha\langle\mathbf{M G}, \mathbf{G}\rangle) \Lambda_{s}$ of $\mathcal{L}_{M}$. Then if we choose $\alpha$ so that the decay factor of this eigenmode is zero, i.e.,

$$
1+(1-\alpha\langle\mathbf{M G}, \mathbf{G}\rangle) \Lambda_{s} \Delta t=0,
$$

or equivalently,

$$
\alpha=\frac{1}{\langle\mathbf{M G}, \mathbf{G}\rangle}\left(1+\frac{1}{\Lambda_{s} \Delta t}\right),
$$

then eigenmode $\mathbf{G}(\mathbf{x})$, which decays the slowest in SOM, becomes decaying the fastest (in fact, this mode is eliminated from the error after a single iteration), while decay rates of the other eigenmodes in the error are unaffected. Thus convergence is improved. In practical situations, the slowest-decaying mode $\mathbf{G}(\mathbf{x})$ and its eigenvalue $\Lambda_{s}$ in SOM are not known. In such cases, if we can choose $\mathbf{G}_{n}$ which resembles the slowest-decaying eigenmode of $\mathrm{SOM}$, then the corresponding eigenvalue $\Lambda_{s}$ can be approximated as the Rayleigh quotient $\Lambda_{s} \approx\left\langle\mathbf{M G}_{n}, \mathcal{L G}_{n}\right\rangle /\left\langle\mathbf{M G}_{n}, \mathbf{G}_{n}\right\rangle$. Substituting this approximation into (8), we get $\alpha$ as given by formula (2). Corresponding to the modified linearization operator (6), the modified iteration method is then MSOM (1).

The above derivation assumed that function $\mathbf{G}_{n}$ is equal or close to the slowest decaying eigenmode of SOM (4). In practical implementations of this method, one does not know priori if the selected $\mathbf{G}_{n}$ meets this criterion. This then may put the effectiveness of this method in question. One may also ask if this modified method can converge at all even with small time steps. Fortunately, the convergence of MSOM is insensitive to the choice of function $\mathbf{G}_{n}$, at least when certain mild conditions are satisfied. Specifically, let us expand function $\mathbf{G}_{n}\left(\mathbf{u}_{n}\right)$ as

$$
\mathbf{G}_{n}=\mathbf{G}_{0}+O\left(\tilde{\mathbf{u}}_{n}\right), \quad \tilde{\mathbf{u}}_{n} \ll 1,
$$

where $\mathbf{G}_{0}$ is $\mathbf{G}_{n}$ 's leading-order term, and $\tilde{\mathbf{u}}_{n}$ is defined by Eq. (10), then we have the following theorem.

Theorem 2 Let Assumption 1 be valid, and $\boldsymbol{L}_{1} \boldsymbol{G}_{0} \not \equiv 0$, then if

$$
\Delta t<\Delta t_{M} \equiv \min \left(-\frac{2}{\Lambda_{\min }}, \frac{1}{\frac{\left\langle\boldsymbol{M} \boldsymbol{G}_{0}, \mathcal{L} \boldsymbol{G}_{0}\right\rangle}{\left\langle\boldsymbol{M} \boldsymbol{G}_{0}, \boldsymbol{G}_{0}\right\rangle}-\Lambda_{\min }}\right),
$$


where $\mathcal{L}$ is given in Eq. (9), $\boldsymbol{M}$ is Hermitian and positive definite, and $\Lambda_{\text {min }}$ is $\mathcal{L}$ 's minimum eigenvalue, then MSOM (1) is guaranteed to converge to the solitary wave $\boldsymbol{u}(\mathbf{x})$ of Eq. (1) if the initial condition is close to $\boldsymbol{u}(\mathbf{x})$.

Proof. We again use the linearization technique to analyze MSOM and prove Theorem 2. Substituting (10) into MSOM (1), we find that the error satisfies the linear iteration equation

$$
\tilde{\mathbf{u}}_{n+1}=\left(1+\Delta t \mathcal{L}_{M}\right) \tilde{\mathbf{u}}_{n},
$$

where

$$
\mathcal{L}_{M} \Psi=\mathcal{L} \Psi-\alpha_{0}\left\langle\mathbf{M G}_{0}, \mathcal{L} \Psi\right\rangle \mathbf{G}_{0}
$$

and

$$
\alpha_{0}=\frac{1}{\left\langle\mathbf{M G}_{0}, \mathbf{G}_{0}\right\rangle}+\frac{1}{\left\langle\mathbf{M G}_{0}, \mathcal{L} \mathbf{G}_{0}\right\rangle \Delta t} .
$$

Note that since $\mathbf{M}$ is Hermitian and positive-definite, $\alpha_{0}$ is real.

Below, we show that all eigenvalues of $\mathcal{L}_{M}$ are real and non-positive, the kernel of $\mathcal{L}_{M}$ is the same as that of $\mathbf{L}_{1}, \mathcal{L}_{M}$ has no square-integrable generalized eigenfunctions at zero eigenvalue, and under the time-step restriction (10), $\left|1+\Lambda_{M} \Delta t\right|<1$ for all non-zero eigenvalues $\Lambda_{M}$ of $\mathcal{L}_{M}$. Then under the assumptions of Theorem 2, MSOM (1) will converge, and Theorem 2 will then be proved.

To proceed, we first write out the eigenvalue problem for operator $\mathcal{L}_{M}$, which is

$$
\mathcal{L} \Psi-\alpha_{0}\left\langle\mathbf{M G}_{0}, \mathcal{L} \Psi\right\rangle \mathbf{G}_{0}=\Lambda_{M} \Psi .
$$

Taking the inner product between this equation and $\mathbf{M} \mathcal{L} \Psi$, and recalling the form (9) of $\mathcal{L}$ and that $\alpha_{0}$ is real and $\mathbf{M}$ Hermitian, we see that eigenvalues $\Lambda_{M}$ are all real. Next, we will analyze the eigenvalue problem (14) by expanding $\Psi$ into eigenfunctions of operator $\mathcal{L}$, a technique which has been used before on other eigenvalue problems $[18,19]$. First, notice from the proof of Theorem 1 that eigenvalues of $\mathcal{L}$ are all real and non-positive, and eigenfunctions of $\mathcal{L}$ form a complete set. Let the discrete and continuous eigenmodes of $\mathcal{L}$ be

$$
\begin{gathered}
\mathcal{L} \psi_{k}(\mathbf{x})=\Lambda_{k} \psi_{k}(\mathbf{x}), \quad \Lambda_{k} \leq 0, \quad k=1, \ldots, m, \\
\mathcal{L} \psi(\mathbf{x} ; \Lambda)=\Lambda \psi(\mathbf{x} ; \Lambda), \quad \Lambda \in I<0,
\end{gathered}
$$

where $m$ is the number of $\mathcal{L}$ 's discrete eigenvalues, $I$ is $\mathcal{L}$ 's continuous spectrum, and the orthogonality conditions among these eigenfunctions are

$$
\left\langle\mathbf{M} \psi_{i}, \psi_{j}\right\rangle=\delta_{i, j},
$$


and

$$
\left\langle\mathbf{M} \psi(\mathbf{x} ; \Lambda), \psi\left(\mathbf{x} ; \Lambda^{\prime}\right)\right\rangle=\delta\left(\Lambda-\Lambda^{\prime}\right) .
$$

Then we expand $\mathbf{G}_{0}$ and $\Psi\left(\mathbf{x} ; \Lambda_{M}\right)$ as

$$
\begin{gathered}
\mathbf{G}_{0}=\sum_{k=1}^{m} c_{k} \psi_{k}(\mathbf{x})+\int_{I} c(\Lambda) \psi(\mathbf{x} ; \Lambda) d \Lambda, \\
\Psi\left(\mathbf{x} ; \Lambda_{M}\right)=\sum_{k=1}^{m} b_{k} \psi_{k}(\mathbf{x})+\int_{I} b(\Lambda) \psi(\mathbf{x} ; \Lambda) d \Lambda .
\end{gathered}
$$

Substituting Eqs. (19) - (20) into the eigenvalue problem (14) and using the orthogonality conditions $(17)-(18)$, one obtains

$$
b_{k}=\frac{\alpha_{0} c_{k}\left\langle\mathbf{M G}_{0}, \mathcal{L} \Psi\right\rangle}{\Lambda_{k}-\Lambda_{M}}, \quad b(\Lambda)=\frac{\alpha_{0} c(\Lambda)\left\langle\mathbf{M G}_{0}, \mathcal{L} \Psi\right\rangle}{\Lambda-\Lambda_{M}} .
$$

Notice that

$$
\begin{gathered}
\left\langle\mathbf{M G}_{0}, \mathcal{L} \Psi\right\rangle=\sum_{k=1}^{m} \Lambda_{k} b_{k} c_{k}^{*}+\int_{I} \Lambda b(\Lambda) c(\Lambda)^{*} d \Lambda \\
=\alpha_{0}\left\langle\mathbf{M G}_{0}, \mathcal{L} \Psi\right\rangle\left(\sum_{k=1}^{m} \frac{\Lambda_{k}\left|c_{k}\right|^{2}}{\Lambda_{k}-\Lambda_{M}}+\int_{I} \frac{\Lambda|c(\Lambda)|^{2}}{\Lambda-\Lambda_{M}} d \Lambda\right),
\end{gathered}
$$

thus we get

$$
\left\langle\mathbf{M G}_{0}, \mathcal{L} \Psi\right\rangle \cdot Q\left(\Lambda_{M}\right)=0
$$

where

$$
Q\left(\Lambda_{M}\right) \equiv \sum_{k=1}^{m} \frac{\Lambda_{k}\left|c_{k}\right|^{2}}{\Lambda_{k}-\Lambda_{M}}+\int_{I} \frac{\Lambda|c(\Lambda)|^{2}}{\Lambda-\Lambda_{M}} d \Lambda-\frac{1}{\alpha_{0}} .
$$

Then the discrete eigenmodes of $\mathcal{L}_{M}$ are such that either

$$
\left\langle\mathbf{M G}_{0}, \mathcal{L} \Psi\right\rangle=0
$$

or

$$
Q\left(\Lambda_{M}\right)=0 .
$$

The continuous eigenvalues of $\mathcal{L}_{M}$ are the same as those of $\mathcal{L}$ since $\mathcal{L}_{M} \rightarrow \mathcal{L}$ as $|\mathbf{x}| \rightarrow \infty$.

We first consider eigenvalues of $\mathcal{L}_{M}$ where condition (25) holds. In this case, Eq. (14) becomes the eigenvalue equation for $\mathcal{L}$, thus these eigenvalues of $\mathcal{L}_{M}$ are also the eigenvalues of $\mathcal{L}$. As a result,

$$
\Lambda_{\min } \leq \Lambda_{M} \leq 0
$$


Next, we consider eigenvalues of $\mathcal{L}_{M}$ which satisfy condition (26). For these eigenvalues, we will show that the following inequality holds:

$$
\min \left(\Lambda_{\min }, \bar{\alpha}_{0}+\Lambda_{\min }\right) \leq \Lambda_{M}<0
$$

where

$$
\bar{\alpha}_{0} \equiv-\alpha_{0}\left\langle\mathbf{M G}_{0}, \mathcal{L} \mathbf{G}_{0}\right\rangle=-\frac{\left\langle\mathbf{M G}_{0}, \mathcal{L} \mathbf{G}_{0}\right\rangle}{\left\langle\mathbf{M G}_{0}, \mathbf{G}_{0}\right\rangle}-\frac{1}{\Delta t} .
$$

To prove the right half of this inequality, recall that $\Lambda_{k} \leq 0$ and $\Lambda \in I<0$, hence for any real number $\Lambda_{M} \geq 0$,

$$
0<\sum_{k=1}^{m} \frac{\Lambda_{k}\left|c_{k}\right|^{2}}{\Lambda_{k}-\Lambda_{M}}+\int_{I} \frac{\Lambda|c(\Lambda)|^{2}}{\Lambda-\Lambda_{M}} d \Lambda \leq \sum_{k=1}^{m}\left|c_{k}\right|^{2}+\int_{I}|c(\Lambda)|^{2} d \Lambda=\left\langle\mathbf{M G}_{0}, \mathbf{G}_{0}\right\rangle
$$

On the other hand,

$$
\frac{1}{\alpha_{0}}=\frac{\left\langle\mathbf{M G}_{0}, \mathbf{G}_{0}\right\rangle}{1+\frac{\left\langle\mathbf{M G}_{0}, \mathbf{G}_{0}\right\rangle}{\left\langle\mathbf{M} \mathbf{G}_{0}, \mathcal{L} \mathbf{G}_{0}\right\rangle \Delta t}}
$$

Since $\mathbf{L}_{1} \mathbf{G}_{0} \neq 0$ by assumption and $\mathbf{M}$ is Hermitian and positive definite, then $\left\langle\mathbf{M G}_{0}, \mathcal{L} \mathbf{G}_{0}\right\rangle<$ 0 . In addition, $\left\langle\mathbf{M G}_{0}, \mathbf{G}_{0}\right\rangle>0$. Thus

$$
\frac{1}{\alpha_{0}}>\left\langle\mathbf{M G}_{0}, \mathbf{G}_{0}\right\rangle \text { or } \frac{1}{\alpha_{0}}<0 .
$$

In view of Eqs. (24), (30) and (32), $Q\left(\Lambda_{M}\right)$ can only have negative roots. Thus, the right half of inequality (28) holds.

In the following, we prove the left half of inequality (28). Notice that

$$
\Lambda_{\min }=\min _{\psi} \frac{\langle\mathbf{M} \psi, \mathcal{L} \psi\rangle}{\langle\mathbf{M} \psi, \psi\rangle}
$$

thus $\bar{\alpha}_{0}+\Lambda_{\min }<0$ in view of Eq. (29) and $\Delta t>0$. Let us rewrite $1 / \alpha_{0}$ as

$$
\frac{1}{\alpha_{0}}=-\frac{\left\langle\mathbf{M G}_{0}, \mathcal{L} \mathbf{G}_{0}\right\rangle}{\bar{\alpha}_{0}}=-\frac{1}{\bar{\alpha}_{0}}\left(\sum_{k=1}^{m} \Lambda_{k}\left|c_{k}\right|^{2}+\int_{I} \Lambda|c(\Lambda)|^{2} d \Lambda\right) .
$$

When this expression is substituted into Eq. (24), we get

$$
Q\left(\Lambda_{M}\right)=\frac{1}{\bar{\alpha}_{0}}\left[\sum_{k=1}^{m} \frac{\left(\Lambda_{k}+\bar{\alpha}_{0}-\Lambda_{M}\right) \Lambda_{k}\left|c_{k}\right|^{2}}{\Lambda_{k}-\Lambda_{M}}+\int_{I} \frac{\left(\Lambda+\bar{\alpha}_{0}-\Lambda_{M}\right) \Lambda|c(\Lambda)|^{2}}{\Lambda-\Lambda_{M}} d \Lambda\right]
$$


When $\Lambda_{M}<\min \left(\Lambda_{\min }, \bar{\alpha}_{0}+\Lambda_{\text {min }}\right)$, all terms inside the square bracket of Eq. (35) are negative, thus $Q\left(\Lambda_{M}\right)$ can not be zero. As a result, the left half of inequality (28) holds.

Due to the two inequalities (27) and (28) on eigenvalues $\Lambda_{M}$ for the two cases (25) and (26), we see that all eigenvalues of $\mathcal{L}_{M}$ are non-positive, and the kernel of $\mathcal{L}_{M}$ is the same as the kernel of $\mathbf{L}_{1}$. In addition, the convergence condition $\left|1+\Lambda_{M} \Delta t\right|<1$ for non-zero eigenvalues $\Lambda_{M}$ of $\mathcal{L}_{M}$ will be satisfied if

$$
\Delta t<-\frac{2}{\Lambda_{\min }}
$$

and

$$
\Delta t<-\frac{2}{\bar{\alpha}_{0}+\Lambda_{\min }} .
$$

Since $\bar{\alpha}_{0}+\Lambda_{\min }<0$ and due to Eq. (29), the second inequality (37) is equivalent to

$$
\Delta t<\frac{1}{\frac{\left\langle\mathbf{M G}_{0}, \mathcal{L} \mathbf{G}_{0}\right\rangle}{\left\langle\mathbf{M G}_{0}, \mathbf{G}_{0}\right\rangle}-\Lambda_{\min }} .
$$

Together with Eq. (36), we find that when $\Delta t$ satisfies the restriction (10), $\left|1+\Lambda_{M} \Delta t\right|<1$ for all non-zero eigenvalues $\Lambda_{M}$ of $\mathcal{L}_{M}$.

Lastly, using similar techniques as employed above, we can readily show that $\mathcal{L}_{M}$ has no generalized eigenfunctions at zero eigenvalue, i.e., equation

$$
\mathcal{L}_{M} \mathbf{F}=\mathbf{u}_{c_{j}}, \quad 1 \leq j \leq s
$$

has no square-integrable solutions $\mathbf{F}$. Here $\mathbf{u}_{c_{j}}$ is in the kernel of $\mathcal{L}_{M}$, i.e. the kernel of $\mathbf{L}_{1}$ (see above and Assumption 1). This rules out the possibility of linear (secular) growth of zero-eigenmodes $\mathbf{u}_{c_{j}}$ in the iteration of the error function $\tilde{\mathbf{u}}_{n}$. This concludes the proof of Theorem 2.

To illustrate the faster convergence of MSOM, we apply MSOM with the choice of (4) to the NLS equation (16), where explicit convergence analysis can be carried out.

Example 3.1 Consider MSOM (1), (4) applied to the solitary wave (17) in the NLS equation (16). For simplicity, we take $M$ as in (18) with $c=\mu=1$. In this case, by inserting $c=1$ into Eq. (22), we find that the eigenvalues of operator $M^{-1} L_{1}$ are

$$
\lambda_{j}=\frac{24}{(2 j+3)^{2}-1}-1, \quad j=0,1,2, \ldots
$$


Hence $\lambda_{0}=2, \lambda_{1}=0, \lambda_{2}=-0.5, \ldots, \lambda_{\infty}=-1$. Eigenvalues of operator $\mathcal{L}$ are

$$
\Lambda=-\lambda_{j}^{2}, \quad j=0,1,2, \ldots
$$

Notice that $M^{-1} L_{1} u=2 u$, hence $G_{0}=u$ is an eigenfunction of $\mathcal{L}$. According to the discussions at the beginning of this section, we find that eigenvalues of $\mathcal{L}_{M}$ are

$$
\begin{gathered}
\Lambda_{M, 0}=-\frac{1}{\Delta t}, \\
\Lambda_{M, j}=-\lambda_{j}^{2}, \quad j=1,2, \ldots
\end{gathered}
$$

Hence the convergence factor $R_{M}$ for MSOM (1) is

$$
R_{M}(\Delta t)=\max \left\{\left|1-\lambda_{2}^{2} \Delta t\right|,\left|1-\lambda_{\infty}^{2} \Delta t\right|\right\}=\max \{|1-0.25 \Delta t|,|1-\Delta t|\} .
$$

It is noted that the zero eigenvalue $\Lambda_{M, 1}$ corresponds to a translational-invariance mode and does not affect the convergence analysis. From Eq. (44), we see that MSOM (1) converges if $\Delta t<\Delta t_{\max }=2$. The fastest convergence is achieved at

$$
\Delta t=\Delta t_{*}=\frac{2}{\lambda_{2}^{2}+\lambda_{\infty}^{2}}=1.6
$$

and the corresponding convergence factor is

$$
R_{M *}=\frac{\lambda_{\infty}^{2}-\lambda_{2}^{2}}{\lambda_{\infty}^{2}+\lambda_{2}^{2}}=0.6
$$

This convergence factor is substantially lower than that of SOM (4) (see Fig. 1), thus MSOM (1), (4) converges much faster. We note in passing that this convergence factor (46) for MSOM is close to those of the optimal Petviashvili method and the optimally accelerated imaginary-time evolution method, which can be calculated to be 0.5 and 0.51 respectively [13].

Remark 3.1 Unlike $\Delta t_{\max }$ in Theorem 1 for SOM, $\Delta t_{M}$ in the time-step restriction (10) is not a sharp bound for convergence of MSOM (1). In practice, $\Delta t$ can often be taken larger than $\Delta t_{M}$, and MSOM still converges. This can be seen clearly in Example 3.1, where $\Lambda_{\text {min }}=-4, G_{0}=u,\left\langle M G_{0}, \mathcal{L} G_{0}\right\rangle /\left\langle M G_{0}, G_{0}\right\rangle=4$, hence $\Delta t_{M}=\frac{1}{8}$. But according to the above explicit calculations, the sharp bound on the time step is $\Delta t_{\max }=2$, much larger than $\Delta t_{M}$. Thus, restriction (10) is sufficient, but not necessary, for the convergence of $\operatorname{MSOM}(1)$. 
Of the two simple choices for $\mathbf{G}_{n}$ given in (4) and (5), $\mathbf{G}_{0}=\mathbf{u}$ for the first choice (4), and $\mathbf{G}_{0}=0$ for the second choice (5). Thus Theorem 2 applies to choice (4), but not to choice (5) since $\mathbf{L}_{1} \mathbf{G}_{0} \equiv 0$ there. The convergence analysis for MSOM with choice (5) is more complicated since the corresponding linearized iteration equation for the error is no longer a one-step iteration, but rather a two-step iteration. However, choice (5) has certain intuitive appeal, as the difference function $\mathbf{u}_{n}-\mathbf{u}_{n-1}$ often resembles the slowest decaying mode in the error [14], thus it makes sense to choose (5). Our numerical experiments have shown that indeed, MSOM with (5) not only converges (when the time step is below a certain threshold), but also converges much faster than the choice (4) in almost all cases. In fact, we will see from various examples in Sec. 6 that MSOM (1) with choice (5) often gives the fastest convergence among schemes proposed in this paper, especially for solitary waves with complicated profiles.

\section{Power-conserving and arbitrary-quantity-conserving squared- operator iteration methods}

In some applications, solitary waves are sought with a pre-specified power (or other quantity such as energy) rather than the propagation constant. For instance, in Bose-Einstein condensation, the number of atoms is often specified, and a solitary wave with that number of atoms (i.e. power in our notations) needs to be computed. In principle, we can still use the aforementioned SOM or MSOM to compute the wave by first continuously varying propagation constants to obtain the power curve, then determining the propagation constant with the pre-specified power, then finally computing the wave again with SOM/MSOM. But that is clearly awkward and not time efficient. A much better way is to design numerical schemes which automatically converge to the solution with the pre-specified quantity. Another example is the linear eigenvalue problem. In this case, the eigenvalues are unknown, thus SOM/MSOM clearly does not apply. If one views the eigenvalues as propagation constants, and requires the eigenfunctions to have a fixed power(norm), then this linear eigenvalue problem becomes the same as a solitary-wave problem with a pre-specified power. To treat this type of problems, one can use the imaginary-time evolution method $[9,10,11,12,13]$, where the solution is normalized at every step to keep the pre-specified power. However, the problem with the imaginary-time evolution method is that it often diverges when the solution crosses zero [13]. Thus new numerical schemes which conserve the power but also have guaranteed convergence need to be constructed. 
In this section, we propose power- (or any other quantity-) conserving squared operator methods, where one specifies the power (or any other quantity) instead of the propagation constant. These methods are in the same spirit as the one presented in [9], but we go much further here. First, we will rigorously prove that these new power-conserving methods converge to any solitary wave as long as the time step is below a certain threshold, and the initial condition is close to that solution - just like SOM/MSOM. This has never been done before. We should point out that this guaranteed convergence for all solitary waves is nontrivial; it is certainly not automatic for an iteration method even if the "squared operator" idea has been incorporated. This guaranteed convergence is possible only when the updating formula for the propagation constants is compatible with the solution updating scheme, in addition to the "squared operator" idea. Second, the methods we will propose use a different acceleration than the acceleration of [9], and hence, as we show in the Appendix, have faster convergence. Thirdly, our methods apply to all types of equations and arbitrary forms of conserved quantities, more general than the method of Ref. [9].

For the ease of presentation, we first consider three special (yet large) classes of equations and construct their power-conserving squared-operator methods (PCSOM). Then we consider the most general case where the wave equations and the pre-specified quantities of the solution are both arbitrary, and construct their quantity-conserving squared-operator methods (QCSOM).

\subsection{The power-conserving squared-operator method for equations with a single propagation constant}

Consider equation (1) of the form

$$
\mathbf{L}_{0} \mathbf{u} \equiv \mathbf{L}_{00} \mathbf{u}-\mu \mathbf{u}=0
$$

Here $\mathbf{u}(\mathbf{x} ; \mu)$ is a real vector solitary wave, and $\mu$ is a real scalar constant (usually called the propagation constant). Solution $\mathbf{u}$ is assumed to exist for continuous ranges of $\mu$ values. Equations of the form (1) include all scalar equations as well as certain vector equations such as vortex equations (2)-(3) in Example 6.1 and the second-harmonic generation system (9)-(10) in Example 6.2. Define the power of solution $\mathbf{u}(\mathbf{x} ; \mu)$ as

$$
P(\mu)=\langle\mathbf{u}(\mathbf{x} ; \mu), \mathbf{u}(\mathbf{x} ; \mu)\rangle,
$$

then we are interested in finding a solution $\mathbf{u}(\mathbf{x} ; \mu)$ whose power has a pre-specified value $P$. Combining ideas of SOM (4) and the imaginary-time evolution method $[9,10,13]$, we 
propose the following new power-conserving squared-operator method (PCSOM):

$$
\mathbf{u}_{n+1}=\left[\frac{P}{\left\langle\hat{\mathbf{u}}_{n+1}, \hat{\mathbf{u}}_{n+1}\right\rangle}\right]^{\frac{1}{2}} \hat{\mathbf{u}}_{n+1}
$$

where

$$
\hat{\mathbf{u}}_{n+1}=\mathbf{u}_{n}-\mathbf{M}^{-1}\left[\mathbf{L}_{1}^{\dagger} \mathbf{M}^{-1} \mathbf{L}_{0} \mathbf{u}-\gamma \mathbf{u}\right]_{\mathbf{u}=\mathbf{u}_{n}, \mu=\mu_{n}} \Delta t,
$$

$\mathbf{M}$ is a positive-definite and Hermitian acceleration operator, and

$$
\gamma=\frac{\left\langle\mathbf{u}, \mathbf{M}^{-1} \mathbf{L}_{1}^{\dagger} \mathbf{M}^{-1} \mathbf{L}_{0} \mathbf{u}\right\rangle}{\left\langle\mathbf{u}, \mathbf{M}^{-1} \mathbf{u}\right\rangle}, \quad \mu_{n}=\left.\frac{\left\langle\mathbf{u}, \mathbf{M}^{-1} \mathbf{L}_{00} \mathbf{u}\right\rangle}{\left\langle\mathbf{u}, \mathbf{M}^{-1} \mathbf{u}\right\rangle}\right|_{\mathbf{u}=\mathbf{u}_{n}} .
$$

The convergence property of this PCSOM is similar to that of the SOM, and is summarized in the following theorem.

Theorem 3 Let Assumption 1 be valid, $\mathbf{u}(\mathbf{x})$ be orthogonal to the kernel of $\mathbf{L}_{1}, P^{\prime}(\mu) \equiv$ $d P(\mu) / d \mu \neq 0$, and define $\Delta t_{\max }$ by $E q$. (8), where $\Lambda_{\text {min }}$ now is the minimum eigenvalue of the iteration operator $\mathcal{L}_{P C}$ defined in Eq. (7) below. Then when $\Delta t<\Delta t_{\text {max }}, P C S O M$ (3)-(5) is guaranteed to converge to the solitary wave $\mathbf{u}(\mathbf{x})$ of $E q$. (1) with power $P$ if the initial condition is close to $\mathbf{u}(\mathbf{x})$. When $\Delta t>\Delta t_{\max }$, PCSOM (3)-(5) diverges.

Proof. As before, we use the linearization method to analyze PCSOM (3)-(5). Substituting Eq. (10) into the scheme and linearizing, we find that the iteration equation for the error $\tilde{\mathbf{u}}_{n}$ is

$$
\tilde{\mathbf{u}}_{n+1}=\tilde{\mathbf{u}}_{n}+\mathcal{L}_{P C} \tilde{\mathbf{u}}_{n} \Delta t
$$

where operator $\mathcal{L}_{P C}$ is

$$
\begin{gathered}
\mathcal{L}_{P C} \Psi=\mathbf{M}^{-1}(\hat{\mathbf{L}} \Psi-\hat{\gamma} \mathbf{u}), \\
\hat{\mathbf{L}} \Psi=-\mathbf{L}_{1}^{\dagger} \mathbf{M}^{-1}\left(\mathbf{L}_{1} \Psi-\beta \mathbf{u}\right),
\end{gathered}
$$

and

$$
\hat{\gamma}=\frac{\left\langle\mathbf{u}, \mathbf{M}^{-1} \hat{\mathbf{L}} \Psi\right\rangle}{\left\langle\mathbf{u}, \mathbf{M}^{-1} \mathbf{u}\right\rangle}, \quad \beta=\frac{\left\langle\mathbf{u}, \mathbf{M}^{-1} \mathbf{L}_{1} \Psi\right\rangle}{\left\langle\mathbf{u}, \mathbf{M}^{-1} \mathbf{u}\right\rangle} .
$$

In addition, due to the power normalization (3), we also have the constraint:

$$
\left\langle\tilde{\mathbf{u}}_{n}, \mathbf{u}\right\rangle=0
$$


Thus it is sufficient to consider eigenfunctions $\Psi(\mathbf{x})$ of operator $\mathcal{L}_{P C}$ in the restricted space $S$ :

$$
S \equiv\{\Psi(\mathbf{x}):\langle\Psi, \mathbf{u}\rangle=0\}
$$

First, we rewrite the operator $\mathcal{L}_{P C}$ as $\mathcal{L}_{P C}=\mathbf{M}^{-1 / 2} \mathcal{L}_{P C H} \mathbf{M}^{1 / 2}$, which defined operator $\mathcal{L}_{P C H}$. Then $\mathcal{L}_{P C}$ and $\mathcal{L}_{P C H}$ are similar, hence having the same eigenvalues. Since we only need to consider eigenfunctions of $\mathcal{L}_{P C}$ which are orthogonal to $\mathbf{u}$, it follows that we only need to consider eigenfunctions of $\mathcal{L}_{P C H}$ which are orthogonal to $\mathbf{M}^{-1 / 2} \mathbf{u}$. In this space, it is easy to check that operator $\mathcal{L}_{P C H}$ is Hermitian. In addition, using the Cauchy-Schwartz inequality, we can verify that $\mathcal{L}_{P C H}$ is also semi-negative definite. Thus, all eigenvalues of $\mathcal{L}_{P C}$ are real and non-positive, and the eigenfunctions of $\mathcal{L}_{P C}$ form a complete set in space $S$. Another way to prove these results is to notice that the operator $\hat{\mathbf{L}}$ is Hermitian and semi-negative definite by inspection and using the Cauchy-Schwartz inequality. Thus operator $\hat{\mathbf{L}} \Psi-\hat{\gamma} \mathbf{u}$ is Hermitian and semi-negative definite in the space $S$. Hence according to the Sylvester inertia law (see, e.g., Theorems 4.5.8 and 7.6.3 in [20]), all eigenvalues of $\mathcal{L}_{P C}$ are real and non-positive, and the eigenfunctions of $\mathcal{L}_{P C}$ form a complete set in space $S$. As a result, under the time-step restriction $\Delta t<\Delta t_{\text {max }}$, the convergence condition $\left|1+\Lambda_{P C} \Delta t\right|<1$ holds for all non-zero eigenvalues $\Lambda_{P C}$ of $\mathcal{L}_{P C}$. On the other hand, if $\Delta t>\Delta t_{\max }, 1+\Lambda_{\min } \Delta t<-1$, hence iterations diverge.

To complete the proof of Theorem 3, it remains to consider the kernel of $\mathcal{L}_{P C}$ in space $S$ and verify that functions $\Psi$ in this kernel do not affect the convergence of the iterations. For these functions, we have

$$
\hat{\mathbf{L}} \Psi-\hat{\gamma} \mathbf{u}=0 .
$$

Taking the inner product between this equation and $\Psi$, and noticing $\Psi \in S$, we get

$$
\langle\hat{\mathbf{L}} \Psi, \Psi\rangle=0 .
$$

Since $\hat{\mathbf{L}}$ is semi-negative definite, from the Cauchy-Schwartz inequality and the condition for its equality to hold, we get

$$
\mathbf{L}_{1} \Psi=\beta \mathbf{u}
$$

On the other hand, differentiating Eq. (1) with respect to $\mu$, we find that

$$
\mathbf{L}_{1} \mathbf{u}_{\mu}=\mathbf{u} .
$$

Thus the solution $\Psi$ of Eq. (14) is equal to $\beta \mathbf{u}_{\mu}$ plus functions in the kernel of $\mathbf{L}_{1}$. Due to the constraint $\Psi \in S$ and recalling our assumptions of $\left\langle\mathbf{u}_{\mu}, \mathbf{u}\right\rangle=\frac{1}{2} P^{\prime}(\mu) \neq 0$ and $\mathbf{u}$ being orthogonal to the kernel of $\mathbf{L}_{1}$, we get $\beta=0$. Thus the kernel of $\mathcal{L}_{P C}$ in space $S$ is the 
same as the kernel of $\mathbf{L}_{1}$. Since this kernel only contains invariance modes by Assumption 1 , it does not affect convergence of the iterations. Combining all the results obtained above, Theorem 3 is then proved.

\subsection{The power-conserving squared-operator method for $K$ equations with $K$ propagation constants}

Next, we consider another class of equations (1) in the form

$$
\mathbf{L}_{\mathbf{0}} \mathbf{u} \equiv \mathbf{L}_{\mathbf{0 0}} \mathbf{u}-\operatorname{diag}\left(\mu_{1}, \ldots, \mu_{K}\right) \mathbf{u}=\mathbf{0},
$$

where $\mathbf{u}(\mathbf{x})=\left[u_{1}, u_{2}, \ldots, u_{K}\right]^{T}$ is a real vector solitary wave, the superscript " $T$ " represents the transpose of a vector, and $\mu_{k}$ 's are real propagation constants which are assumed to be independent of each other. The key feature of this case is that the number of independent propagation constants $\mu_{k}$ is equal to the number of components in the vector solution $\mathbf{u}(\mathbf{x})$. Defining the powers of individual components $u_{k}(\mathbf{x})$ of the solution as

$$
P_{k} \equiv\left\langle u_{k}, u_{k}\right\rangle, \quad 1 \leq k \leq K,
$$

and introducing the following $K \times K$ matrix

$$
\mathbf{D}_{K \times K} \equiv\left(\frac{\partial P_{i}}{\partial \mu_{j}}\right)
$$

then the PCSOM for Eq. (16) we propose is

$$
u_{k, n+1}=\left[\frac{P_{k}}{\left\langle\hat{u}_{k, n+1}, \hat{u}_{k, n+1}\right\rangle}\right]^{\frac{1}{2}} \hat{u}_{k, n+1}, \quad 1 \leq k \leq K,
$$

where

$$
\begin{gathered}
\hat{\mathbf{u}}_{n+1}=\mathbf{u}_{n}-\mathbf{M}^{-1}\left\{\mathbf{L}_{1}^{\dagger} \mathbf{M}^{-1} \mathbf{L}_{0} \mathbf{u}-\operatorname{diag}\left(\gamma_{1}, \ldots, \gamma_{K}\right) \mathbf{u}\right\}_{\mathbf{u}=\mathbf{u}_{n}, \mu_{k}=\mu_{k, n}} \Delta t, \\
\gamma_{k}=\frac{\left\langle u_{k},\left[\mathbf{M}^{-1} \mathbf{L}_{1}^{\dagger} \mathbf{M}^{-1} \mathbf{L}_{0} \mathbf{u}\right]_{k}\right\rangle}{\left\langle u_{k}, M_{k}^{-1} u_{k}\right\rangle}, \quad \mu_{k, n}=\left.\frac{\left\langle u_{k},\left[\mathbf{M}^{-1} \mathbf{L}_{\mathbf{0 0}} \mathbf{u}\right]_{k}\right\rangle}{\left\langle u_{k}, M_{k}^{-1} u_{k}\right\rangle}\right|_{\mathbf{u}=\mathbf{u}_{n}} .
\end{gathered}
$$

Here $[\cdot]_{k}$ represents the $k$-th component of the vector inside the bracket, $[\cdot]_{k, n}$ represents the $n$-th iteration of the $k$-th component of the vector inside the bracket, and $\mathbf{M} \equiv$ $\operatorname{diag}\left(M_{1}, M_{2}, \ldots, M_{K}\right)$ is a positive definite and Hermitian acceleration operator. 
The convergence properties of this PCSOM for Eq. (16) are similar to those of the previous PCSOM, and they are summarized in the following theorem.

Theorem 4 Let all $\mu_{k}$ 's be independent, Assumption 1 be valid, $\mathbf{u}(\mathbf{x})$ be orthogonal to the kernel of $\mathbf{L}_{\mathbf{1}}, \operatorname{det}(\boldsymbol{D}) \neq 0$, and define $\Delta t_{\text {max }}$ by $E q$. (8), where $\Lambda_{\text {min }}$ here is the minimum eigenvalue of the operator $\mathcal{L}_{P C V}$ defined as

$$
\mathcal{L}_{P C V} \Psi=\mathbf{M}^{-1}\left\{\hat{\mathbf{L}}_{V} \Psi-\operatorname{diag}\left(\hat{\gamma}_{1}, \ldots, \hat{\gamma_{K}}\right) \boldsymbol{u}\right\}
$$

where

$$
\hat{\mathbf{L}}_{V} \Psi=-\mathbf{L}_{1}^{\dagger} \mathbf{M}^{-1}\left[\mathbf{L}_{1} \Psi-\operatorname{diag}\left(\beta_{1}, \ldots, \beta_{K}\right) \boldsymbol{u}\right]
$$

and

$$
\hat{\gamma_{k}}=\frac{\left\langle u_{k},\left[\mathbf{M}^{-1} \hat{\mathbf{L}}_{V} \Psi\right]_{k}\right\rangle}{\left\langle u_{k}, M_{k}^{-1} u_{k}\right\rangle}, \quad \beta_{k}=\frac{\left\langle u_{k},\left[\mathbf{M}^{-1} \mathbf{L}_{1} \Psi\right]_{k}\right\rangle}{\left\langle u_{k}, M_{k}^{-1} u_{k}\right\rangle} .
$$

Then when $\Delta t<\Delta t_{\max }$, PCSOM (19)-(21) is guaranteed to converge to the solitary wave $\boldsymbol{u}(\mathbf{x})$ of Eq. (16) with component powers being $\left[P_{1}, \ldots, P_{k}\right]^{T}$, if the initial condition is close to $\boldsymbol{u}(\mathbf{x})$. When $\Delta t>\Delta t_{\max }, P C S O M$ (19)-(21) diverges.

Proof. The proof of this theorem is analogous to that of Theorem 3. By the linearization analysis, we find that the error of the iterated function satisfies the equation

$$
\tilde{\mathbf{u}}_{n+1}=\tilde{\mathbf{u}}_{n}+\mathcal{L}_{P C V} \tilde{\mathbf{u}}_{n} \Delta t,
$$

where operator $\mathcal{L}_{P C V}$ is as defined in Eq. (22). The power normalization (19) implies that it suffices to consider the eigenfunctions $\Psi$ of $\mathcal{L}_{P C V}$ satisfying the following orthogonality conditions:

$$
\left\langle\Psi_{k}, u_{k}\right\rangle=0, \quad k=1, \ldots, K .
$$

Similar to the operator $\mathcal{L}_{P C}$ in the proof of Theorem 3, we can show that in the space of functions satisfying the above orthogonality conditions, all eigenvalues of $\mathcal{L}_{P C V}$ are real and non-positive, and all its eigenfunctions form a complete set. The main difference between the previous operator $\mathcal{L}_{P C}$ and $\mathcal{L}_{P C V}$ here is in their kernels. The kernel of operator $\mathcal{L}_{P C V}$ contains functions $\Psi$ where $\mathcal{L}_{P C V} \Psi=0$. Similar to the case in Theorem 3, we can show that

$$
\mathbf{L}_{1} \Psi=\operatorname{diag}\left(\beta_{1}, \beta_{2}, \ldots, \beta_{K}\right) \mathbf{u} .
$$

Differentiating Eq. (16) with respect to $\mu_{k}$, we get

$$
\mathbf{L}_{1} \mathbf{u}_{\mu_{k}}=\left(0, \ldots, u_{k}, \ldots, 0\right)^{T}, \quad k=1, \ldots, K .
$$


Hence the solution of Eq. (27) is

$$
\Psi=\sum_{k=1}^{K} \beta_{k} \mathbf{u}_{\mu_{k}}
$$

plus the homogeneous solution of Eq. (27). Substituting this equation into the orthogonality conditions (26), and recalling the assumptions of Theorem 4, we get

$$
\mathbf{D} \cdot \vec{\beta}=0,
$$

where matrix $\mathbf{D}$ is defined in Eq. (18), and $\vec{\beta}=\left(\beta_{1}, \beta_{2}, \ldots, \beta_{K}\right)^{T}$. Since $\operatorname{det}(\mathbf{D}) \neq 0$ by assumption, the solution to Eq. (30) is $\vec{\beta}=\overrightarrow{0}$. Hence the kernel of $\mathcal{L}_{P C V}$ satisfying the orthogonality conditions (26) is the same as the kernel of $\mathbf{L}_{1}$. Thus, Theorem 4 is proved.

The PCSOMs (3)-(5) and (19)-(21) have been presented above as separate cases because they have simple forms and also apply to many equations that arise frequently in applications. For example, the method of Section 4.1 applies to linearly coupled nonlinear Schrödinger equations, while the method of Section 4.2 applies to nonlinearly coupled ones; see also Examples 6.1-6.3 below. A PCSOM would also result for a more general case where in Eq. (1) of the form (16), the propagation constants $\mu_{k}$ 's can be separated into several independent groups of equal $\mu_{k}$ 's. An example of such a system of equations can be found, e.g., in [21]. In this case, the PCSOM can be constructed by combining the two PCSOMs (3)-(5) and (19)-(21). Here we group together the solution components $u_{k}$ 's whose corresponding propagation constants $\mu_{k}$ 's are the same. These groups form sub-vectors in the whole solution vector $\mathbf{u}(\mathbf{x})$. Then the PCSOM for this more general equation is analogous to PCSOM (19)-(21), except that $u_{k}$ is replaced by each sub-vector of the solution, and $P_{k}$ replaced by that sub-vector's power. The convergence properties of this more general PCSOM are similar to those in Theorems 3 and 4, and will not be elaborated here.

\subsection{The squared-operator method with general quadratic conserved quan- tities}

In this subsection, we present the PCSOM for the case where the conserved quantities are not restricted to simple powers (as in Secs. 4.1 and 4.2). Rather, they can be more general quadratic quantities of the solutions (i.e. linear combinations of powers of individual components of the vector solitary wave). This case includes as particular cases the PCSOMs presented in Sections 4.1 and 4.2. We chose to present it separately from those two methods because its form is more complex than theirs, while the situations where neither of those 
two simpler methods could apply are relatively rare. One example of such a situation is the system of coherent three-wave interactions in a quadratic medium [22, 23], which has only two conserved quantities which are linear combinations of the three wave powers (these conservation laws are known as Manley-Rowe relations).

The class of problems described above can be formulated mathematically as follows:

$$
\mathbf{L}_{0} \mathbf{u} \equiv \mathbf{L}_{00} \mathbf{u}-\frac{\delta \mathbf{Q}}{\delta \mathbf{u}} \vec{\mu}=0
$$

and

$$
Q_{j}(\mathbf{u}) \equiv \sum_{l=1}^{K} q_{j k} P_{k}=C_{j}, \quad j=1, \ldots, r .
$$

Here $\mathbf{u}(\mathbf{x})=\left[u_{1}, u_{2}, \ldots, u_{K}\right]^{T}$ is a vector solitary wave, $\delta / \delta \mathbf{u}=\left[\delta / \delta u_{1}, \ldots, \delta / \delta u_{K}\right]^{T}$ is the functional derivative, $\vec{\mu}=\left(\mu_{1}, \ldots, \mu_{r}\right)^{T}$ is the vector of $r$ linearly independent propagation constants $(1 \leq r \leq K), P_{k}$ are defined in (17), $q_{j k}$ are constants, and $C_{j}$ are the specified values of the quadratic conserved quantities $Q_{j}$. All variables and constants involved are real-valued. Without any loss of generality, we assume that the $r \times K$ matrix of the coefficients $q_{j k}$ is in the reduced echelon form such that

$$
q_{i j}= \begin{cases}0, & i>j \\ 1, & i=j .\end{cases}
$$

Then, using the terminology of textbooks in introductory Linear Algebra, we refer to powers $\left\{P_{k}\right\}_{k=r+1}^{K}$ and $\left\{P_{k}\right\}_{k=1}^{r}$ as "independent" and "dependent" powers, respectively.

The PCSOM that we propose for Eqs. (31)-(32) has the following form:

$$
\begin{aligned}
u_{k, n+1} & =\left[\frac{C_{k}-\sum_{l=r+1}^{K} q_{k l} \hat{P}_{l}}{\left\langle\hat{u}_{k, n+1}, \hat{u}_{k, n+1}\right\rangle}\right]^{1 / 2} \hat{u}_{k, n+1}, \quad 1 \leq k \leq r, \\
\hat{\mathbf{u}}_{n+1} & =\mathbf{u}_{n}-\mathbf{M}^{-1}\left[\mathbf{L}_{1}^{\dagger} \mathbf{M}^{-1} \mathbf{L}_{0} \mathbf{u}-\mathbf{B} \vec{\gamma}_{n}\right]_{\mathbf{u}=\mathbf{u}_{n}, \vec{\mu}=\vec{\mu}_{n}} \Delta t,
\end{aligned}
$$

where the notations for $\mathbf{L}_{1}, \mathbf{M}$, and the subscripts are the same as before, $\hat{P}_{k}=\left\langle\hat{u}_{k, n+1}, \hat{u}_{k, n+1}\right\rangle$,

$$
\begin{gathered}
\mathbf{B} \equiv \frac{\delta \mathbf{Q}}{\delta \mathbf{u}}, \\
\vec{\gamma}_{n}=\left.\left\langle\mathbf{B}, \mathbf{M}^{-1} \mathbf{B}\right\rangle^{-1}\left\langle\mathbf{B}, \mathbf{M}^{-1} \mathbf{L}_{1}^{\dagger} \mathbf{M}^{-1} \mathbf{L}_{0} \mathbf{u}\right\rangle\right|_{\mathbf{u}=\mathbf{u}_{n}},
\end{gathered}
$$


and

$$
\vec{\mu}_{n}=\left.\left\langle\mathbf{B}, \mathbf{M}^{-1} \mathbf{B}\right\rangle^{-1}\left\langle\mathbf{B}, \mathbf{M}^{-1} \mathbf{L}_{00} \mathbf{u}\right\rangle\right|_{\mathbf{u}=\mathbf{u}_{n}} .
$$

The power-normalization step (34) is written with the account of the reduced echelon form (33) of the matrix $\left(q_{j k}\right)$. Note that this step affects only the "dependent" powers, while the "independent" ones do not need to be normalized.

Convergence conditions of the PCSOM (34)-(38) are similar to those of the PCSOMs described above. Introducing the notation

$$
\hat{\mathbf{D}}_{r \times r} \equiv\left(\frac{\partial Q_{i}}{\partial \mu_{j}}\right),
$$

then convergence conditions of this PCSOM is summarized in the following theorem.

Theorem 5 Let Assumption 1 be valid, all columns of the matrix $\mathbf{B}$ in (36) be orthogonal to the kernel of $\mathbf{L}_{\mathbf{1}}$, and $\operatorname{det}(\hat{\mathbf{D}}) \neq 0$. Also, let $\Delta t_{\text {max }}$ be given by $E q$. (8) where $\Lambda_{\text {min }}$ now is the minimum eigenvalue of the operator $\mathcal{L}_{P C G}$ defined in Eq. (40) below. Then for $\Delta t<\Delta t_{\text {max }}$, the PCSOM (34)-(38) is guaranteed to converge to the solitary wave $\boldsymbol{u}(\mathbf{x})$ of Eq. (31) which satisfies the constraints (32), provided that the initial condition is close to $\boldsymbol{u}(\mathbf{x})$. When $\Delta t>\Delta t_{\max }$, the PCSOM (34)-(38) diverges.

The proof of this theorem follows the lines of Theorems 3 and 4, thus we will only sketch it below, emphasizing the differences from the proofs of the latter theorems.

Proof. Linearizing Eq. (35), and noticing that the power normalization step (34) does not affect the linearized equations (as in Sections 4.1 and 4.2), we find that the error satisfies the iteration equation similar to (6), except that the iteration operator now becomes

$$
\mathcal{L}_{P C G} \Psi=\mathbf{M}^{-1}\left(\hat{\mathbf{L}}_{P C G} \Psi-\mathbf{B}\left\langle\mathbf{B}, \mathbf{M}^{-1} \mathbf{B}\right\rangle^{-1}\left\langle\mathbf{B}, \mathbf{M}^{-1} \hat{\mathbf{L}}_{P C G} \Psi\right\rangle\right)
$$

where

$$
\hat{\mathbf{L}}_{P C G} \Psi=-\mathbf{L}_{1}^{\dagger} \mathbf{M}^{-1}\left(\mathbf{L}_{1} \Psi-\mathbf{B}\left\langle\mathbf{B}, \mathbf{M}^{-1} \mathbf{B}\right\rangle^{-1}\left\langle\mathbf{B}, \mathbf{M}^{-1} \mathbf{L}_{1} \Psi\right\rangle\right) .
$$

The eigenfunctions $\Psi$ satisfy the orthogonality relation

$$
\left\langle B_{j}, \Psi\right\rangle=0, \quad j=1, \ldots, r,
$$

with $B_{j}$ being the $j$-th column of $\mathbf{B}$. These conditions follow from the quantities $Q_{j}$ being conserved and from the relation (36) between $\mathbf{B}$ and $Q_{j}$ 's. 
The operator $\hat{\mathbf{L}}_{P C G}$ is Hermitian. In addition, using the generalized Cauchy-Schwartz inequality for any matrix functions of $\mathbf{F}_{1}$ and $\mathbf{F}_{2}$ :

$$
\left\langle\mathbf{F}_{1}, \mathbf{F}_{1}\right\rangle \geq\left\langle\mathbf{F}_{1}, \mathbf{F}_{2}\right\rangle\left\langle\mathbf{F}_{2}, \mathbf{F}_{2}\right\rangle^{-1}\left\langle\mathbf{F}_{2}, \mathbf{F}_{1}\right\rangle
$$

one can verify that $\hat{\mathbf{L}}_{P C G}$ is semi-negative definite. Then following the lines of the proof of Theorem 3, one then finds that in the space of functions satisfying the orthogonality conditions (42), all eigenvalues of $\mathcal{L}_{P C G}$ are real and non-positive, and all its eigenfunctions form a complete set.

Then it remains to consider the kernel of $\mathcal{L}_{P C G}$, which satisfies the equation

$$
\hat{\mathbf{L}}_{P C G} \Psi-\mathbf{B}\left\langle\mathbf{B}, \mathbf{M}^{-1} \mathbf{B}\right\rangle^{-1}\left\langle\mathbf{B}, \mathbf{M}^{-1} \hat{\mathbf{L}}_{P C G} \Psi\right\rangle=0
$$

and show that the eigenfunctions of this kernel can only be those in the kernel of $\mathbf{L}_{1}$ and thus would not affect the convergence of this PCSOM in view of our assumptions. To that end, we take the inner product between Eq. (44) and $\Psi$, use the orthogonality relations (42), then recall that operator $\hat{\mathbf{L}}_{P C G}$ is semi-negative definite, and finally notice that the Cauchy-Schwartz inequality (43) becomes an equality if and only if $\mathbf{F}_{1}$ and $\mathbf{F}_{2}$ are linearly related by a constant matrix. This yields

$$
\mathbf{L}_{1} \Psi=\mathbf{B} \vec{\beta}
$$

where $\vec{\beta}=\left(\beta_{1}, \ldots, \beta_{r}\right)^{T}$ is a constant vector. Noticing the relations obtained by differentiating Eq. (31) with respect to $\mu_{j}(1 \leq j \leq r)$, we see, similarly to $(29)$, that the solution to Eq. (45) is

$$
\Psi=\sum_{j=1}^{r} \beta_{j} \mathbf{u}_{\mu_{j}}
$$

plus functions in the kernel of $\mathbf{L}_{1}$. Substituting this solution into (42) and recalling that by the assumptions of Theorem 5 , columns of $\mathbf{B}$ are orthogonal to the kernel of $\mathbf{L}_{1}$ and $\operatorname{det}(\hat{\mathbf{D}}) \neq 0$, we find that $\vec{\beta}=0$. Thus the kernel of $\mathcal{L}_{P C G}$ is the same as that of $\mathbf{L}_{1}$. Summarizing all these results, Theorem 5 is then proved.

\subsection{The squared-operator method for general equations with arbitrary conserved quantities}

The most general case is probably that the equations depend on the propagation constants $\mu_{k}$ 's in a general (but linear) way, and the specified conserved quantities of the solutions 
are not restricted to powers or linear combinations of powers, but can be arbitrary. These equations and constraints can be written in the general form

$$
\mathbf{L}_{0} \mathbf{u} \equiv \mathbf{L}_{00} \mathbf{u}-\mathbf{B}(\mathbf{u}) \vec{\mu}=0
$$

and

$$
Q_{j}(\mathbf{u})=C_{j}, \quad j=1, \ldots, r,
$$

where $\mu=\left(\mu_{1}, \ldots, \mu_{r}\right)^{T}$ is the vector of all independent propagation constants, $\mathbf{B}$ is a general matrix function of $\mathbf{u}$, and $Q_{j}(\mathbf{u})$ are arbitrary functionals which are pre-specified. All quantities involved are real-valued. This system (47)-(48) generalizes the system (31)(32) in the previous subsection in two significant ways. First, the functionals $Q_{j}$ are not restricted to the quadratic form (32) of $\mathbf{u}$, but are allowed to be arbitrary functionals. For instance, one can seek a solution with a prescribed value of the Hamiltonian. Second, matrix B is not restricted to the special form (36) as in Eq. (31), but is allowed to be arbitrary functions of $\mathbf{u}$. In this general case, counterparts of the power normalization step (34) become impossible. Thus, for the solution to have the pre-specified quantities (48), new ideas are necessary. Our idea is to replace the power normalization step with adding new terms into the iteration step (35) in such a way that when iterations converge, the final solution is guaranteed to meet the constraints (48). Our proposed scheme for this general case with arbitrary conserved quantities, which we denote QCSOM, is

$$
\mathbf{u}_{n+1}=\mathbf{u}_{n}-\mathbf{M}^{-1}\left[\mathbf{L}_{1}^{\dagger} \mathbf{M}^{-1} \mathbf{L}_{0} \mathbf{u}+h \sum_{j=1}^{r}\left(Q_{j}(\mathbf{u})-C_{j}\right) \frac{\delta Q_{j}}{\delta \mathbf{u}}\right]_{\mathbf{u}=\mathbf{u}_{n}, \vec{\mu}=\vec{\mu}_{n}} \Delta t,
$$

where $\vec{\mu}_{n}$ is defined by Eq. (38), and $h>0$ is a user-specified free scheme parameter whose purpose will be explained after the proof of Theorem 6 . The idea behind this scheme is that instead of minimizing the functional appearing on the right-hand side of Eq. (6) in Section 2, one minimizes a modified functional which equals the one from Eq. (6) plus additional terms $\frac{1}{2} \sum\left[Q_{j}(\mathbf{u})-C_{j}\right]^{2}$. The acceleration (with the operator $\mathbf{M}^{-1}$ ) of this scheme is performed in the same way as the acceleration of (5).

On the convergence of this QCSOM for Eqs. (47) and (48), we have the following theorem, which is very similar to Theorem 5 of the previous subsection.

Theorem 6 Let Assumption 1 be valid, $\delta Q_{j} / \delta \mathbf{u}(j=1, \ldots r)$ be orthogonal to the kernel of $\mathbf{L}_{\mathbf{1}}, \operatorname{det}(\hat{\mathbf{D}}) \neq 0$, and define $\Delta t_{\max }$ by $E q .(8)$, where $\Lambda_{\text {min }}$ here is the minimum eigenvalue of the operator $\mathcal{L}_{Q C}$ defined in Eq. (50) below, then when $\Delta t<\Delta t_{\text {max }}, Q C S O M$ (49) is 
guaranteed to converge to the solitary wave $\boldsymbol{u}(\mathbf{x})$ of Eq. (47) which satisfies the constraints (48), if the initial condition is close to $\boldsymbol{u}(\mathbf{x})$. When $\Delta t>\Delta t_{\max }, Q C S O M$ (49) diverges.

Here $\operatorname{det}(\hat{\mathbf{D}})$ is as defined in Eq. (39), but $Q_{j}$ is an arbitrary function of $\mathbf{u}$ now.

The proof of this theorem is also very similar to that of Theorem 5, thus we will only highlight its differences from the proof of the latter Theorem. Namely, the principal difference is that due to the absence of the normalization step in QCSOM (49), (38), there appears to be no counterpart of the orthogonality condition (42), which played a critical role in the proof in Section 4.3. However, as we will see, a direct counterpart of that condition will arise from different considerations.

Proof. Linearizing the QCSOM, we find that the error satisfies the iteration equation similar to (6), except that the iteration operator now becomes

$$
\mathcal{L}_{Q C} \Psi=\mathbf{M}^{-1}\left(\hat{\mathbf{L}}_{P C G} \Psi-h \sum_{j=1}^{r}\left\langle\frac{\delta Q_{j}}{\delta \mathbf{u}}, \Psi\right\rangle \frac{\delta Q_{j}}{\delta \mathbf{u}}\right)
$$

where $\hat{\mathbf{L}}_{P C G}$ is defined by Eq. (41) of Section 4.3. The operator $\mathcal{L}_{Q C}$ can be rewritten as $\mathcal{L}_{Q C}=\mathbf{M}^{-1 / 2} \mathcal{L}_{Q C H} \mathbf{M}^{1 / 2}$. It is easy to check that $\mathcal{L}_{Q C H}$ is Hermitian. Using the generalized Cauchy-Schwartz inequality (43), we can also verify that $\mathcal{L}_{Q C H}$ is semi-negative definite. Thus all eigenvalues of $\mathcal{L}_{Q C}$ are real and non-positive, and all eigenfunctions of $\mathcal{L}_{Q C}$ form a complete set. The kernel of $\mathcal{L}_{Q C}$ satisfies the equation

$$
\hat{\mathbf{L}}_{P C G} \Psi-h \sum_{j=1}^{r}\left\langle\frac{\delta Q_{j}}{\delta \mathbf{u}}, \Psi\right\rangle \frac{\delta Q_{j}}{\delta \mathbf{u}}=0 .
$$

Notice that both terms in the above equation are Hermitian and semi-negative definite operators, thus when taking the inner product between this equation and $\Psi$, we find that $\Psi$ in the kernel of $\mathcal{L}_{Q C}$ satisfies Eq. (45) as well as the orthogonality relations

$$
\left\langle\frac{\delta Q_{j}}{\delta \mathbf{u}}, \Psi\right\rangle=0, \quad j=1, \ldots, r .
$$

These relations are the counterparts of the orthogonality relations (42) of the previous subsection. Then the proof of this theorem is completed in exactly the same way as the proof of Theorem 5 .

Now we explain the reason for introducing the free parameter $h$ into the scheme (49). Our experience shows that in some cases (especially when the conserved quantities $C_{j}$ are large), 
the $\delta Q_{j} / \delta \mathbf{u}$ terms in Eq. (50) with $h=1$ cause operator $\mathcal{L}_{Q C}$ 's minimum eigenvalue $\Lambda_{\text {min }}$ to be large negative - much larger than that of the operator $-\mathbf{M}^{-1} \hat{\mathbf{L}}_{Q C}$ in magnitude. This forces us to take very small time steps $\Delta t$ (see Theorem 6), which severely slows down the convergence speed. When this happens, our strategy is to introduce a small parameter $h$ into the scheme (49). The idea is to reduce the effect of the $\delta Q_{j} / \delta \mathbf{u}$ terms on the operator $\mathcal{L}_{Q C}$, and make its minimum eigenvalue close (or equal) to that of the operator $-\mathbf{M}^{-1} \hat{\mathbf{L}}_{Q C}$. Hence the fast convergence of the scheme will be restored. This parameter $h$ needs to be positive so that the relevant terms in Eq. (50) are semi-negative definite.

To conclude this section, we point out that the general structures of the PCSOM (34)-(38) and QCSOM (49) can also be used to construct the imaginary-time evolution methods for the case when, in the above notations, $1<r<K$. To our knowledge, such a case was not considered in Refs. [9, 10, 12], where imaginary-time evolution methods for vector equations were reported. Of course, the corresponding imaginary-time evolution methods, unlike the PCSOM (34)-(38) and QCSOM (49), will not be universally-convergent.

\section{Squared-operator iteration methods for isolated solitary waves}

In many dissipative wave systems such as the Ginsburg-Landau equation, solitary waves exist only when the propagation constants in the equation take discrete (isolated) values. We call the corresponding solutions isolated solitary waves. For these isolated solutions, the propagation constants or powers of the solutions are unknown and need to be computed together with the solitary wave, thus the numerical schemes discussed in previous sections do not apply. In this section, we propose squared-operator iteration methods for isolated solitary waves.

For simplicity, we consider the case where a vector isolated solitary wave exists when a single propagation constant takes on discrete values (other cases can be easily extended). The equation for the solitary wave can be written as (1), but the solution $\mathbf{u}(\mathbf{x})$ now exists only at an unknown discrete $\mu$ value. We propose the following squared-operator iteration method for these isolated solitary waves (SOMI):

$$
\begin{gathered}
\mathbf{u}_{n+1}=\mathbf{u}_{n}-\left[\mathbf{M}^{-1} \mathbf{L}_{1}^{\dagger} \mathbf{M}^{-1} \mathbf{L}_{0} \mathbf{u}\right]_{\mathbf{u}=\mathbf{u}_{n}, \mu=\mu_{n}} \Delta t \\
\mu_{n+1}=\mu_{n}+\left.\left\langle\mathbf{u}, \mathbf{M}^{-1} \mathbf{L}_{0} \mathbf{u}\right\rangle\right|_{\mathbf{u}=\mathbf{u}_{n}, \mu=\mu_{n}} \Delta t
\end{gathered}
$$


where $\mathbf{L}_{1}$ is the linear operator as defined in Eq. (2), and $\mathbf{M}$ is a positive-definite and Hermitian acceleration operator. Linearizing this method around the isolated solitary wave, we get the iteration equation for the error $\left(\tilde{\mathbf{u}}_{n}, \tilde{\mu}_{n}\right)$ as

$$
\left(\begin{array}{c}
\tilde{\mathbf{u}}_{n+1} \\
\tilde{\mu}_{n+1}
\end{array}\right)=\left(1+\Delta t \mathcal{L}_{I}\right)\left(\begin{array}{c}
\tilde{\mathbf{u}}_{n} \\
\tilde{\mu}_{n}
\end{array}\right)
$$

where

$$
\mathcal{L}_{I}\left(\begin{array}{c}
\tilde{\mathbf{u}}_{n} \\
\tilde{\mu}_{n}
\end{array}\right) \equiv\left(\begin{array}{c}
-\mathbf{M}^{-1} \mathbf{L}_{1}^{\dagger} \mathbf{M}^{-1}\left(\mathbf{L}_{1} \tilde{\mathbf{u}}_{n}-\tilde{\mu}_{n} \mathbf{u}\right) \\
\left\langle\mathbf{u}, \mathbf{M}^{-1}\left(\mathbf{L}_{1} \tilde{\mathbf{u}}_{n}-\tilde{\mu}_{n} \mathbf{u}\right)\right\rangle
\end{array}\right) .
$$

It is easy to check that operator $\mathcal{L}_{I}$ can be written as $\operatorname{diag}\left(\mathbf{M}^{-1 / 2}, 1\right) \mathcal{L}_{I H} \operatorname{diag}\left(\mathbf{M}^{1 / 2}, 1\right)$, where $\mathcal{L}_{I H}$ is Hermitian and semi-negative definite. Thus all eigenvalues of $\mathcal{L}_{I}$ are real and non-positive, and all its eigenfunctions form a complete set. The kernel of $\mathcal{L}_{I}$ contains functions $[\Phi(\mathbf{x}), 0]^{T}$ with $\Phi(\mathbf{x})$ in the kernel of $\mathbf{L}_{1}$, as well as functions $[\mathbf{F}(\mathbf{x}), 1]^{T}$ where $\mathbf{F}(\mathbf{x})$ satisfies the equation

$$
\mathbf{L}_{1} \mathbf{F}=\mathbf{u}
$$

and must be bounded. Assuming that $\mathbf{u}$ is not orthogonal to the kernel of operator $\mathbf{L}_{1}^{\dagger}$ (which is the generic case), then Eq. (5) has no bounded solution, hence the kernel of $\mathcal{L}_{I}$ only contains the invariance modes $[\Phi(\mathbf{x}), 0]^{T}$. Then under Assumption 1, SOMI (1)-(2) will converge if $\Delta t<-2 / \Lambda_{\min }$ and diverge if $\Delta t>-2 / \Lambda_{\min }$, where $\Lambda_{\min }$ is the minimum eigenvalue of operator $\mathcal{L}_{I}$.

Following the motivation for MSOM (1) in Sec. 3, we can construct the modified squared operator method for isolated solitons in Eq. (1) as follows (MSOMI):

$$
\mathbf{u}_{n+1}=\mathbf{u}_{n}+\left[-\mathbf{M}^{-1} \mathbf{L}_{1}^{\dagger} \mathbf{M}^{-1} \mathbf{L}_{0} \mathbf{u}-\alpha_{n} \theta_{n} \mathbf{G}_{n}\right]_{\mathbf{u}=\mathbf{u}_{n}, \mu=\mu_{n}} \Delta t
$$

and

$$
\mu_{n+1}=\mu_{n}+\left[\left\langle\mathbf{u}, \mathbf{M}^{-1} \mathbf{L}_{0} \mathbf{u}\right\rangle-\alpha_{n} \theta_{n} H_{n}\right]_{\mathbf{u}=\mathbf{u}_{n}, \mu=\mu_{n}} \Delta t
$$

where

$$
\begin{gathered}
\alpha_{n}=\frac{1}{\left\langle\mathbf{M G}_{n}, \mathbf{G}_{n}\right\rangle+H_{n}^{2}}-\frac{1}{\left\langle\left(\mathbf{L}_{1} \mathbf{G}_{n}-H_{n} \mathbf{u}\right), \mathbf{M}^{-1}\left(\mathbf{L}_{1} \mathbf{G}_{n}-H_{n} \mathbf{u}\right)\right\rangle \Delta t}, \\
\theta_{n}=-\left\langle\mathbf{L}_{1} \mathbf{G}_{n}-H_{n} \mathbf{u}, \mathbf{M}^{-1} \mathbf{L}_{0} \mathbf{u}\right\rangle,
\end{gathered}
$$

and $\left(\mathbf{G}_{n}, H_{n}\right)$ are functions specified by the user. A good choice, which is a counterpart of Eq. (5), is

$$
\mathbf{G}_{n}=\mathbf{e}_{n} \equiv \mathbf{u}_{n}-\mathbf{u}_{n-1}, \quad H_{n}=\mu_{n}-\mu_{n-1} .
$$

We can show that under similar conditions as in Theorem 2 and the assumption below Eq. (5), this MSOMI also converges for all isolated solitary waves. 


\section{Examples of applications of squared-operator iteration meth- ods}

In this section, we consider various examples of physical interest to illustrate the performances of the proposed schemes. Most of these examples are two-dimensional, thus the shooting method can not work. For some of the examples such as Examples 1 (b,c) and 5, other numerical methods such as the Petviashvili method and the imaginary-time evolution method can not work either.

Example 6.1 Consider solitary waves in the two-dimensional NLS equation with a periodic potential,

$$
U_{x x}+U_{y y}-V_{0}\left(\sin ^{2} x+\sin ^{2} y\right) U+\sigma|U|^{2} U=-\mu U,
$$

which arises in nonlinear light propagation in photonic lattices and Bose-Einstein condensation in optical lattices. Here $\mu$ is the real propagation constant, and $U$ is a complex solution in general. Writing $U$ into its real and imaginary components, $U(x, y)=u(x, y)+i v(x, y)$, we get equations for the real functions $u$ and $v$ as

$$
\begin{aligned}
& u_{x x}+u_{y y}-V_{0}\left(\sin ^{2} x+\sin ^{2} y\right) u+\sigma\left(u^{2}+v^{2}\right) u=-\mu u, \\
& v_{x x}+v_{y y}-V_{0}\left(\sin ^{2} x+\sin ^{2} y\right) v+\sigma\left(u^{2}+v^{2}\right) v=-\mu v .
\end{aligned}
$$

This system is rotationally invariant, i.e., if $(u, v)^{T}$ is a solution, so is

$$
\left(\begin{array}{cc}
\cos \theta & -\sin \theta \\
\sin \theta & \cos \theta
\end{array}\right)\left(\begin{array}{l}
u \\
v
\end{array}\right),
$$

where $\theta$ is the angle of rotation (which is constant). This invariance, in Eq. (1), corresponds to $U \rightarrow U e^{i \theta}$. This rotational invariance induces an eigenmode $(-v, u)^{T}$ in the kernel of $\mathbf{L}_{1}$. Clearly, this eigenmode is orthogonal to the solution $(u, v)^{T}$, satisfying the orthogonality condition in Theorem 3. The kernel of $\mathbf{L}_{1}$ does not contain other eigenfunctions (at least in the generic case) since there are no other invariances in this system.

Solitary waves in Eq. (1) exist only inside the bandgaps of the system. Thus, the bandgap information is needed first. The bandgap diagram at various values of $V_{0}$ is displayed in Fig. 2(a). For illustration purpose, we fix $V_{0}=6$, and determine the solitary waves in different bandgaps below. 
(a) Vortex solitons in the semi-infinite bandgap under focusing nonlinearity:

For focusing nonlinearity, $\sigma=1$. In this case, Eq. (1) admits various types of real and complex solitary-wave solutions in every bandgap of the system [24, 25, 26]. Here we determine a vortex-soliton solution at $\mu=3(P=14.6004)$, which is marked by letter 'a' in the semi-infinite bandgap of Fig. 2(a). This solution is complex valued with a non-trivial phase structure, and it is displayed in Fig. 2(c, d). Similar solutions have been reported theoretically in [24] before, and have since been experimentally observed $[27,28]$. To determine this solution, we apply the SOM (4), MSOM (1), (5), and PCSOM (3)-(5) on Eqs. (2)-(3), starting from the initial condition

$U(x, y)=1.7\left(e^{-x^{2}-y^{2}}+e^{-(x-\pi)^{2}-y^{2}+i \pi / 2}+e^{-(x-\pi)^{2}-(y-\pi)^{2}+i \pi}+e^{-x^{2}-(y-\pi)^{2}+3 i \pi / 2}\right)$.

In addition, we choose the acceleration operator $\mathbf{M}$ as

$$
\mathbf{M}=\left(c-\partial_{x x}-\partial_{y y}\right) \operatorname{diag}(1,1) .
$$

The spatial derivatives as well as $\mathbf{M}^{-1}$ are computed by the discrete Fourier transform (i.e., by the pseudo-spectral method). The computational domain is $-6 \pi \leq x, y \leq 6 \pi$, discretized in each dimension by 256 grid points. It should be noted that the size of the computational domain and the number of grid points have very little effect on the convergence speed; they mainly affect the spatial accuracy of the solution. For these three schemes, we found that the optimal (or nearly optimal) parameters are $(c, \Delta t)=(3.7,0.8)$ for SOM and PCSOM, and $(c, \Delta t)=(3.8,0.6)$ for MSOM. At these choices of parameters, the error diagrams versus the number of iterations are displayed in Fig. 2(b). Here the error is defined as the difference between successive iteration functions:

$$
e_{n}=\sqrt{\left\langle U_{n}-U_{n-1}, U_{n}-U_{n-1}\right\rangle} \text {. }
$$

We see that all three schemes converge rather quickly. The convergence speeds of SOM and PCSOM are almost the same, but MSOM converges much faster. It should be noted that the amount of computations in one iteration is different for these three methods, with the ratio roughly of $1: 1.7: 2$ for SOM, PCSOM, and MSOM. When this factor is also considered, we conclude MSOM converges the fastest, with SOM second, and PCSOM third.

(b) Solitons in the first bandgap under defocusing nonlinearity:

Next, we consider solutions in the first bandgap (between the first and second Bloch bands) under defocusing nonlinearity $(\sigma=-1)$. For this purpose, we pick $\mu=5$, 
marked by letter 'b' in the first bandgap of Fig. 2(a). At this point, Eq. (1) admits a real-valued gap soliton, which is displayed in Fig. 3(a). Similar solutions have been reported in $[29,30]$ before. To determine this solution, we apply the SOM (4), MSOM (1), (5), and PCSOM (3)-(5) on Eq. (1), starting from the initial condition

$$
U(x, y)=1.15 \operatorname{sech}\left(x^{2}+y^{2}\right) \cos (x) \cos (y) .
$$

We take $\mathbf{M}$ as

$$
\mathbf{M}=c-\partial_{x x}-\partial_{y y} .
$$

The computational domain is $-5 \pi \leq x, y \leq 5 \pi$, discretized in each dimension by 128 grid points. For these three schemes, we found that the optimal (or nearly optimal) parameters are $(c, \Delta t)=(1.8,0.6)$ for SOM and PCSOM, and $(c, \Delta t)=(2.9,1.7)$ for MSOM. At these choices of parameters, the error diagrams versus the number of iterations are displayed in Fig. 3(b). Similar to the vortex soliton in Fig. 2, we find that the convergence speeds of SOM and PCSOM are almost the same, but MSOM converges much faster.

(c) Vortex solitons in the second bandgap under defocusing nonlinearity:

We now determine vortex solitons in the second bandgap under defocusing nonlinearity $(\sigma=-1)$. For this purpose, we pick $\mu=9.4$, marked by letter 'c' in the second bandgap of Fig. 2(a). At this point, a vortex soliton with distinctive amplitude and phase distributions exists (see Fig. 4(a, b)). It is noted that this vortex soliton is not of the type reported in $[30,26]$, which lie in the first bandgap (in our notations). To our knowledge, this type of soliton has never been reported before in the literature. To determine this solution, we apply the SOM (4), MSOM (1), (5), and PCSOM (3)-(5) on Eqs. (2)-(3). The initial condition is the vortex solution of these equations at a different value $\mu=9.42$, which we in turn obtained by the continuation method from a small-amplitude solution near the edge of the bandgap. The acceleration operator $\mathbf{M}$ is the same as (5). The computational domain is $-10 \pi \leq x, y \leq 10 \pi$, discretized in each dimension by 256 grid points. For these three schemes, we found that the optimal (or nearly optimal) parameters are $(c, \Delta t)=(4.2,1.7)$ for SOM and PCSOM, and $(c, \Delta t)=(4,3.1)$ for MSOM. At these choices of parameters, the error diagrams versus the number of iterations are displayed in Fig. 4(b). In this case, MSOM is again the fastest. However, unlike the above two cases, PCSOM converges much slower than SOM now.

Example 6.2 Consider the following system arising from a second-harmonic generation 
(SHG) model,

$$
\begin{gathered}
u_{x x}+u_{y y}+u v=\mu u, \\
\frac{1}{2}\left[v_{x x}+5 v_{y y}+\frac{1}{2} u^{2}-v\right]=\mu v .
\end{gathered}
$$

Solutions of similar systems have been considered in a number of studies; see, e.g., a recent paper [31] and references therein. Note that in the original reduction from the SHG model, the right hand side of the $v$-equation is usually $2 \mu v$. But in order to cast the equations into the form (1), we have divided the $v$-equation by 2 , so that we work with Eq. (10) instead. Here we deliberately make the $v_{x x}$ and $v_{y y}$ coefficients different, so that the radial symmetry is broken, hence this system is not reducible to a radially symmetric (and hence essentially one-dimensional) problem. At $\mu=0.1$, this system admits a solitary wave with total power $P=\langle u, u\rangle+\langle v, v\rangle=47.3744$, which is displayed in Fig. 5(a, b). We take the initial condition as

$$
u(x, y)=\operatorname{sech}\left(0.35 \sqrt{x^{2}+y^{2}}\right), \quad v=0.3 \operatorname{sech}\left(0.5 \sqrt{x^{2}+y^{2}}\right) .
$$

The acceleration operator $\mathbf{M}$ is taken as

$$
\mathbf{M}=\operatorname{diag}\left[\mu-\partial_{x x}-\partial_{y y}, \mu+\frac{1}{2}-\frac{1}{2} \partial_{x x}-\frac{5}{2} \partial_{y y}\right],
$$

where the choice of constants $\mu$ and $\mu+\frac{1}{2}$ is motivated by our earlier results on optimal accelerations for imaginary-time evolution methods [13]. The computational domain is $-25 \leq x, y, \leq 25$, and the number of grid points along each dimension is 64 . The iteration results of SOM (4), MSOM (1), (5) and PCSOM (3)-(5) at optimal (or nearly optimal) $\Delta t$ values $0.37,0.59$, and 0.63 respectively are displayed in Fig. 5(c). Again, MSOM delivers the best performance, and PCSOM is the slowest.

Example 6.3 The next example is the coupled two-dimensional NLS equations with saturable nonlinearity,

$$
\begin{aligned}
& u_{x x}+u_{y y}+\frac{u^{2}+v^{2}}{1+s\left(u^{2}+v^{2}\right)} u=\mu_{1} u, \\
& v_{x x}+v_{y y}+\frac{u^{2}+v^{2}}{1+s\left(u^{2}+v^{2}\right)} v=\mu_{2} v,
\end{aligned}
$$

which has been studied, e.g., in $[9,32,33]$. Here $s$ is the saturation constant. At $s=$ $0.5, \mu=1, \mu_{2}=0.5\left(P_{1}=85.3884, P_{2}=29.1751\right)$, this system admits a solution whose $u$-component is single-humped, but its $v$-component is a dipole state. This solution is 
displayed in Fig. 6(a, b). Note that this solution is not radially symmetric, thus is not reducible to a one-dimensional problem. Taking the initial condition as

$$
u(x, y)=3 e^{-0.2 r^{2}}, \quad v(x, y)=1.5 r e^{-0.2 r^{2}} \cos \theta,
$$

where $(r, \theta)$ are the polar coordinates, the acceleration operator $\mathbf{M}$ as

$$
\mathbf{M}=\operatorname{diag}\left[\mu_{1}-\partial_{x x}-\partial_{y y}, \mu_{2}-\partial_{x x}-\partial_{y y}\right],
$$

the computational domain as $-12 \leq x, y, \leq 12$, and the number of grid points along the two dimensions as 64, the iteration results of SOM (4), MSOM (1), (5) and PCSOM (19)-(21) at optimal $\Delta t$ values 1.9, 2.65, and 1.85 are displayed in Fig. 6(c). As in (12) above, the choice of the constants $\mu_{1}$ and $\mu_{2}$ in the acceleration operator (16) is also motivated by our previous studies on the accelerated imaginary-time evolution method [13]. Again, MSOM delivers the best performance, and PCSOM is the slowest.

Example 6.4 The next example is intended to compare the performances of PCSOM (34)(38) and QCSOM (49). This example comes from the three-wave interaction system [22, 23] and has the form

$$
\begin{gathered}
u_{x x}+u_{y y}+v w=\mu_{1} u, \\
v_{x x}+v_{y y}+u w=\mu_{2} v, \\
w_{x x}+w_{y y}+u v=\left(\mu_{1}+\mu_{2}\right) w,
\end{gathered}
$$

where $u, v$ and $w$ are real functions, and $\mu_{1}$ and $\mu_{2}$ are propagation constants. At $\mu_{1}=0.5$ and $\mu_{2}=1$, this system has a radially symmetric solution displayed in Fig. 7(a), and

$$
Q_{1} \equiv\langle u, u\rangle+\langle w, w\rangle=66.3096, \quad Q_{2} \equiv\langle v, v\rangle+\langle w, w\rangle=47.2667 .
$$

Here we want to determine this solution with the pre-specified quantities $Q_{1}$ and $Q_{2}$ as above. Note that this problem is of the form (31)-(32) and (47)-(48), but not of the form (1) or (16). Taking the initial condition as

$$
u(x, y)=2.5 \operatorname{sech} 0.8 r, \quad v(x, y)=2.2 \operatorname{sech} 0.8 r, \quad w(x, y)=1.9 \operatorname{sech} 0.8 r,
$$

where $r=\sqrt{x^{2}+y^{2}}$, the acceleration operator $\mathbf{M}$ as

$$
\mathbf{M}=\operatorname{diag}\left[\mu_{1}-\partial_{x x}-\partial_{y y}, \mu_{2}-\partial_{x x}-\partial_{y y}, \mu_{1}+\mu_{2}-\partial_{x x}-\partial_{y y}\right],
$$

the computational domain as $-15 \leq x, y, \leq 15$, the number of grid points along the two dimensions as 64, and the parameter $h$ in the QCSOM as $h=0.01$, the iteration results of 
PCSOM (34)-(38) and QCSOM (49) at the (same) optimal value $\Delta t=0.49$ are displayed in Fig. 7(b). This figure shows that the QCSOM converges slightly slower than the PCSOM. However, it is noted that each QCSOM iteration involves less computations than the PCSOM, thus we conclude that the performances of PCSOM and QCSOM are comparable.

Example 6.5 The Ginzburg-Landau equation is of the form

$$
i \Phi_{t}+\left(1-\gamma_{1} i\right) \Phi_{x x}-i \gamma_{0} \Phi+|\Phi|^{2} \Phi=0
$$

where $\gamma_{0}$ is the damping/pumping coefficient (when $\gamma_{0}$ is negative/positive). We seek solitary waves in this equation of the form

$$
\Phi(x, t)=U(x) e^{i \mu t},
$$

where $\mu$ is a real propagation constant, then function $U(x)$ satisfies the equation

$$
\left(1-\gamma_{1} i\right) U_{x x}-i \gamma_{0} U+|U|^{2} U=\mu U
$$

If $\gamma_{0}$ or $\gamma_{1}$ is non-zero, then solitary waves in this equation are always complex-valued, and they can only exist at isolated propagation constants. When $\gamma_{0}=0.3$ and $\gamma_{1}=1$, the solitary wave, which exists at the discrete value $\mu=1.2369$, is plotted in Fig. 8(a). Writing this equation into two real-valued equations for $[\operatorname{Re}(U), \operatorname{Im}(U)]$, and applying SOMI (1)-(2) or MSOMI (6)-(10) with $M=c-\partial_{x x}$ and initial conditions $u_{0}(x)=1.6 \operatorname{sech}(x), \mu_{0}=1.2$, we can obtain this isolated solitary wave. At optimal scheme parameters $(c, \Delta t)=(1.6,0.3)$ for SOMI and $(c, \Delta t)=(1.4,0.12)$ for MSOMI, the error diagram is displayed in Fig. $8(\mathrm{~b})$. Here the error is defined as

$$
e_{n}=\sqrt{\left\langle u_{n}-u_{n-1}, u_{n}-u_{n-1}\right\rangle}+\left|\mu_{n}-\mu_{n-1}\right| .
$$

We see that MSOMI converges much faster than SOMI, which is consistent with previous numerical experiments.

We should point out that in this example, since $\gamma_{0}>0$, the soliton we obtained is unstable (because the zero background is unstable). So this soliton can not be obtained by simulating the Ginzburg-Landau equation (23). However, our proposed method SOMI/MSOMI can produce this unstable solution quite easily.

\section{Summary}

In this paper, we have developed three iteration methods - the squared operator method (SOM), the modified squared operator method (MSOM), and the power(or any quantity)- 
conserving squared operator method (PCSOM/QCSOM), for computing solitary waves in general nonlinear wave equations. The solitary waves can exist at either continuous or discrete propagation constants. These numerical methods are based on iterating new differential equations whose linearization operators are squares of those for the original equations. We proved that all these methods are guaranteed to converge to all types of solitary waves as long as the time step in the iteration schemes is below a certain threshold value. Due to the use of acceleration techniques, these methods are fast converging. Since these methods are compatible with the pseudo-spectral method, their spatial accuracy is exponentially high. Furthermore, these methods can treat problems in arbitrary dimensions with little change in the programming, and they are very easy to implement. To test the relative performances of these methods, we have applied them to various solitary wave problems of physical interest, such as higher-gap vortex solitons in the two-dimensional nonlinear Schrödinger equations with periodic potentials and isolated solitons in Ginzburg-Landau equations. We found that MSOM delivers the best performance among all the methods proposed.

Even though MSOM delivers the best performance, SOM and PCSOM/QCSOM have their own advantages as well. For instance, SOM is simpler to implement. PCSOM/QCSOM would be advantageous if the problem at hand specifies the power or other conserved quantity of the solution rather than the propagation constants. In addition, PCSOM/QCSOM works for linear eigenvalue problems (by setting the linear eigenfunction to have a fixed norm), while SOM and MSOM do not. In some cases, SOM or PCSOM is more tolerant to the choice of initial conditions, i.e., they converge for a larger range of initial conditions than MSOM. Thus all the methods developed in this paper can be useful for different purposes, and the reader can pick them judiciously depending on the problem at hand.

It is noted that these methods can deliver good performance even with suboptimal choices of scheme parameters. But how to get the best performance out of these schemes (i.e., how to find optimal scheme parameters) is still an important open question. For the Petviashvili method, conditions for optimal or nearly optimal convergence have been studied in $[6,14]$. For the accelerated imaginary-time evolution method, optimal acceleration parameters have been obtained for a large class of equations [13]. Such results can help us to select the scheme parameters for the squared-operator methods in this paper (as we have done in Examples 6.2-6.4). But a rigorous and comprehensive study on optimal scheme parameters for the squared-operator methods proposed in this paper is a non-trivial issue and will be left for future studies. 


\section{Acknowledgment}

Part of this work was done when J.Y. was visiting Zhou Pei-Yuan Center for Applied Mathematics at Tsinghua University. The hospitality of the Center was appreciated. The work of J.Y. was supported in part by AFOSR under grant USAF 9550-05-1-0379, and the work of T.I.L. was supported in part by NSF under grant DMS-0507429.

\section{Appendix: Families of squared-operator methods}

The squared operator methods proposed in this paper were based on the operator $\mathcal{L}$ given in Eq. (9). It turns out that one can construct a family of squared operator methods which contain the methods in this paper as particular cases. Consider the following squared operator iteration method for Eq. (1):

$$
\mathbf{u}_{n+1}=\mathbf{u}_{n}-\left[\mathbf{M}^{-a} \mathbf{L}_{1}^{\dagger} \mathbf{M}^{-b} \mathbf{L}_{0} \mathbf{u}\right]_{\mathbf{u}=\mathbf{u}_{n}} \Delta t
$$

where $\mathbf{M}$ is a positive definite Hermitian operator, and $a$ and $b$ are arbitrary constants. The linearized equation of this method for the error $\tilde{\mathbf{u}}_{n}$ is still Eq. (11), except that $\mathcal{L}$ is replaced by

$$
\mathcal{L}_{f} \equiv-\mathbf{M}^{-a} \mathbf{L}_{1}^{\dagger} \mathbf{M}^{-b} \mathbf{L}_{1}
$$

now. This operator can be rewritten as

$$
\mathcal{L}_{f}=-\mathbf{M}^{-a / 2}\left(\mathbf{M}^{-b / 2} \mathbf{L}_{1} \mathbf{M}^{-a / 2}\right)^{\dagger}\left(\mathbf{M}^{-b / 2} \mathbf{L}_{1} \mathbf{M}^{-a / 2}\right) \mathbf{M}^{a / 2},
$$

thus its eigenvalues are clearly all non-positive. Repeating the proof in Sec. 2, we can readily show that the SOM (A.1) is guaranteed to converge if $\Delta t<-2 / \Lambda_{\min }$, where $\Lambda_{\min }$ is the minimum eigenvalue of operator $\mathcal{L}_{f}$. If we choose $a=1$ and $b=1$, then method (A.1) becomes SOM (4). For the family of SOMs (A.1), the corresponding MSOMs, PCSOMs, as well as methods for isolated solitary waves can be readily constructed. Below we illustrate such a construction for a particular choice of $a$ and $b$. Namely, we consider the member of family (A.1) with $a=0$ and $b=2$, whose implementation of each iteration requires slightly fewer operations than the implementation of method (4) in the main text. In this case, the corresponding squared operator methods are listed below.

- SOM for Eq. (1):

$$
\mathbf{u}_{n+1}=\mathbf{u}_{n}-\left[\mathbf{L}_{1}^{\dagger} \mathbf{M}^{-2} \mathbf{L}_{0} \mathbf{u}\right]_{\mathbf{u}=\mathbf{u}_{n}} \Delta t
$$


- MSOM for Eq. (1):

$$
\mathbf{u}_{n+1}=\mathbf{u}_{n}-\left[\mathbf{L}_{1}^{\dagger} \mathbf{M}^{-2} \mathbf{L}_{0} \mathbf{u}-\alpha_{n}\left\langle\mathbf{G}_{n}, \mathbf{L}_{1}^{\dagger} \mathbf{M}^{-2} \mathbf{L}_{0} \mathbf{u}\right\rangle \mathbf{G}_{n}\right]_{\mathbf{u}=\mathbf{u}_{n}} \Delta t
$$

where

$$
\alpha_{n}=\frac{1}{\left\langle\mathbf{G}_{n}, \mathbf{G}_{n}\right\rangle}-\frac{1}{\left\langle\mathbf{M}^{-1} \mathbf{L}_{1} \mathbf{G}_{n}, \mathbf{M}^{-1} \mathbf{L}_{1} \mathbf{G}_{n}\right\rangle \Delta t},
$$

and $\mathbf{G}_{n}$ is a user-specified function such as (5).

- PCSOM for Eq. (1):

$$
\begin{gathered}
\mathbf{u}_{n+1}=\left[\frac{P}{\left\langle\hat{\mathbf{u}}_{n+1}, \hat{\mathbf{u}}_{n+1}\right\rangle}\right]^{\frac{1}{2}} \hat{\mathbf{u}}_{n+1}, \\
\hat{\mathbf{u}}_{n+1}=\mathbf{u}_{n}-\left[\mathbf{L}_{1}^{\dagger} \mathbf{M}^{-2} \mathbf{L}_{0} \mathbf{u}-\gamma \mathbf{u}\right]_{\mathbf{u}=\mathbf{u}_{n}, \mu=\mu_{n}} \Delta t
\end{gathered}
$$

and

$$
\gamma=\frac{\left\langle\mathbf{u}, \mathbf{L}_{1}^{\dagger} \mathbf{M}^{-2} \mathbf{L}_{0} \mathbf{u}\right\rangle}{\langle\mathbf{u}, \mathbf{u}\rangle}, \quad \mu_{n}=\left.\frac{\left\langle\mathbf{u}, \mathbf{M}^{-2} \mathbf{L}_{00} \mathbf{u}\right\rangle}{\left\langle\mathbf{u}, \mathbf{M}^{-2} \mathbf{u}\right\rangle}\right|_{\mathbf{u}=\mathbf{u}_{n}} .
$$

We note that for this power-conserving scheme, the $\gamma$ term in Eq. (A.8) can be dropped due to the presence of the power normalization step (A.7). It is easy to check that the reduced scheme has the same linearized iteration operator as the original one above, thus possesses the same convergence properties. However, this can not be done for the PCSOM (3)-(5) in the main text if $\mathbf{M} \neq 1$. The other forms of the PCSOM for the cases (16) and (31)-(32) can be similarly written down.

- QCSOM for Eqs. (47)-(48):

$$
\mathbf{u}_{n+1}=\mathbf{u}_{n}-\left[\mathbf{L}_{1}^{\dagger} \mathbf{M}^{-2} \mathbf{L}_{0} \mathbf{u}+h \sum_{j=1}^{r}\left(Q_{j}(\mathbf{u})-C_{j}\right) \frac{\delta Q_{j}}{\delta \mathbf{u}}\right]_{\mathbf{u}=\mathbf{u}_{n}, \vec{\mu}=\vec{\mu}_{n}} \Delta t
$$

where

$$
\vec{\mu}_{n}=\left.\left\langle\mathbf{B}, \mathbf{M}^{-2} \mathbf{B}\right\rangle^{-1}\left\langle\mathbf{B}, \mathbf{M}^{-2} \mathbf{L}_{00} \mathbf{u}\right\rangle\right|_{\mathbf{u}=\mathbf{u}_{n}} .
$$

- SOMI for isolated solitary waves in Eq. (1):

$$
\begin{aligned}
& \mathbf{u}_{n+1}=\mathbf{u}_{n}-\left[\mathbf{L}_{1}^{\dagger} \mathbf{M}^{-2} \mathbf{L}_{0} \mathbf{u}\right]_{\mathbf{u}=\mathbf{u}_{n}, \mu=\mu_{n}} \Delta t, \\
& \mu_{n+1}=\mu_{n}+\left.\left\langle\mathbf{u}, \mathbf{M}^{-2} \mathbf{L}_{0} \mathbf{u}\right\rangle\right|_{\mathbf{u}=\mathbf{u}_{n}, \mu=\mu_{n}} \Delta t,
\end{aligned}
$$


- MSOMI for isolated solitary waves in Eq. (1):

$$
\begin{aligned}
& \mathbf{u}_{n+1}=\mathbf{u}_{n}+\left[-\mathbf{L}_{1}^{\dagger} \mathbf{M}^{-2} \mathbf{L}_{0} \mathbf{u}-\alpha_{n} \theta_{n} \mathbf{G}_{n}\right]_{\mathbf{u}=\mathbf{u}_{n}, \mu=\mu_{n}} \Delta t, \\
& \mu_{n+1}=\mu_{n}+\left[\left\langle\mathbf{u}, \mathbf{M}^{-2} \mathbf{L}_{0} \mathbf{u}\right\rangle-\alpha_{n} \theta_{n} H_{n}\right]_{\mathbf{u}=\mathbf{u}_{n}, \mu=\mu_{n}} \Delta t,
\end{aligned}
$$

where

$$
\begin{gathered}
\alpha_{n}=\frac{1}{\left\langle\mathbf{G}_{n}, \mathbf{G}_{n}\right\rangle+H_{n}^{2}}-\frac{1}{\left\langle\mathbf{M}^{-1}\left(\mathbf{L}_{1} \mathbf{G}_{n}-H_{n} \mathbf{u}\right), \mathbf{M}^{-1}\left(\mathbf{L}_{1} \mathbf{G}_{n}-H_{n} \mathbf{u}\right)\right\rangle \Delta t}, \\
\theta_{n}=-\left\langle\mathbf{G}_{n}, \mathbf{L}_{1}^{\dagger} \mathbf{M}^{-2} \mathbf{L}_{0} \mathbf{u}\right\rangle+\left\langle H_{n} \mathbf{u}, \mathbf{M}^{-2} \mathbf{L}_{0} \mathbf{u}\right\rangle,
\end{gathered}
$$

and $\left(\mathbf{G}_{n}, H_{n}\right)$ are user specified functions such as (10).

All these methods can be shown to have similar convergence properties as their counterparts in the main text.

As we have already noted, in all the methods presented in this Appendix starting with Eq. (A.4), each iteration involves a little less computations than their counterparts in the methods presented in the main text. However, we did not advocate for these methods for two reasons. First, we can show that the convergence of these methods is always slower than that of their counterparts in the text when $\mathbf{L}_{1}$ is Hermitian. In such a case, using Theorem 5.6.9 in [20], we can show that $\Lambda_{\min } / \Lambda_{\max }$ is smaller for $\mathcal{L}$ than it is for $\mathcal{L}_{f}$, hence SOM (4) converges faster than its counterpart (A.4) in view of Eq. (15). Second, for the squared-operator methods in the main text, we can carry out explicit convergence analysis on some familiar examples such as the NLS equation. This would not be possible for the methods considered in this Appendix. Overall, our numerical testing shows that the squared-operator methods presented in the main text give the best performance among the family of methods (A.1) with other choices of $a$ and $b$.

\section{References}

[1] J.P. Boyd, "Why Newton's Method is Hard for Travelling Waves: Small Denominators, KAM Theory, Arnold's Linear Fourier Problem, Non-Uniqueness, Constraints and Erratic Failure," preprint. 
[2] J. Yang, "Internal oscillations and instability characteristics of $(2+1)$ dimensional solitons in a saturable nonlinear medium," Phys. Rev. E. 66, 026601 (2002).

[3] M. Mitchell, M. Segev, T.H. Coskun and D.N. Christodoulides, "Theory of selftrapped spatially incoherent light beams", Phys. Rev. Lett. 79, 49904993 (1997).

[4] P.G. Kevrekidis, K.O. Rasmussen, and A.R. Bishop, "Localized excitations and their thresholds," Phys. Rev. E 61, 4652 (2000).

[5] V. I. Petviashvili, "Equation of an extraordinary soliton," Plasma Physics, 2, 469-472, (1976).

[6] D.E. Pelinovsky and Yu. A. Stepanyants, "Convergence of Petviashvili's iteration method for numerical approximation of stationary solutions of nonlinear wave equations," SIAM J. Numer. Anal. 42, 1110 (2004).

[7] Z.H. Musslimani and J. Yang, "Localization of light in a two-dimensional periodic structure," J. Opt. Soc. Am. B 21, 973-981 (2004).

[8] M.J. Ablowitz and Z.H. Musslimani, "Spectral renormalization method for computing self-localized solutions to nonlinear systems," Opt. Lett. 30, 2140-2142 (2005).

[9] J.J. Garcia-Ripoll and V.M. Perez-Garcia, "Optimizing Schrödinger functionals using Sobolev gradients: Applications to quantum mechanics and nonlinear optics," SIAM J. Sci. Comput. 23, 1316 (2001).

[10] W. Bao and Q. Du, "Computing the ground state solution of Bose-Einstein condensates by a normalized gradient flow," SIAM J. Sci. Comput. 25, 1674-1697 (2004).

[11] W. Bao, "Ground states and dynamics of multi-component Bose-Einstein condensates," Multiscale Modeling and Simulation, 2, 210-236 (2004).

[12] V.S. Shchesnovich and S.B. Cavalcanti, "Rayleigh functional for nonlinear systems," available at http://www.arXiv.org, Preprint nlin.PS/0411033.

[13] J. Yang and T.I. Lakoba, "Convergence and acceleration of imaginary-time evolution methods for solitary waves in arbitrary spatial dimensions," submitted.

[14] T.I. Lakoba and J. Yang, "A generalized Petviashvili iteration method for scalar and vector Hamiltonian equations with arbitrary form of nonlinearity," submitted. 
[15] J. Yang and Z. Chen, "Defect solitons in photonic lattices," Phys. Rev. E 73, 026609 (2006).

[16] L.D. Landau and E.M. Lifshitz, Quantum Mechanics: non-relativistic theory, 3 ed., Butterworth-Heinemann, Oxford (1977).

[17] J. Yang and D.J. Benney, "Some properties of nonlinear wave systems," Stud. Appl. Math. 96, 111-139 (1996).

[18] N. G. Vakhitov and A. A. Kolokolov, "Stationary solutions of the wave equation in the medium with nonlinearity saturation," Izv. Vyssh. Uchebn. Zaved. Radiofiz. 16, 1020 (1973) [Radiophys. Quantum Electron. 16, 783 (1973)].

[19] W.D. Lakin, R. Mathon, and A. Nachman, "Buckling and vibration of a rotating spoke," J. Engineering Math. 12, 193-206 (1978).

[20] R. Horn and C. Johnson, Matrix Analysis, Cambridge University Press, New York, 1991.

[21] T.I. Lakoba, D.J. Kaup, B.A. Malomed, "Solitons in nonlinear directional coupler with two orthogonal polarizations," Phys. Rev. E 55, 6107 (1997).

[22] Yu. N. Karamzin, A. P. Sukhorukov, and T. S. Filipchuk, "New class of coupled solutions in dispersive media with quadratic nonlinearity," Vestn. Mosk. Univ., Ser. 3: Fiz., Astron. 19, 91-98 (1978) (in Russian) [English translation: Moscow Univ. Phys. Bull. 33, 73 (1978)].

[23] A.V. Buryak, P. Di Trapani, D.V. Skryabin and S. Trillo, "Optical solitons due to quadratic nonlinearities: from basic physics to futuristic applications," Physics Reports, 370, Issue 2, 63-235 (2002).

[24] J. Yang and Z. Musslimani, "Fundamental and vortex solitons in a two-dimensional optical lattice," Opt. Lett. 28, 2094 (2003).

[25] J. Yang, I. Makasyuk, A. Bezryadina, and Z. Chen, "Dipole and quadrupole solitons in optically-induced two-dimensional photonic lattices: theory and experiment." Stud. Appl. Math. 113, 389 (2004).

[26] G. Bartal, O. Manela, O. Cohen, J.W. Fleischer, and M. Segev, "Observation of second-band vortex solitons in 2D photonic lattices", Phys. Rev. Lett. 95, 053904 $(2005)$. 
[27] D.N. Neshev, T.J. Alexander, E.A. Ostrovskaya, Y.S. Kivshar, H. Martin, Z. Chen, Observation of Discrete Vortex Solitons in Optically Induced Photonic Lattices. Phys. Rev. Lett. 92, 123903 (2004).

[28] J.W. Fleischer, G. Bartal, O. Cohen, O. Manela, M. Segev, J. Hudock, D.N. Christodoulides, Observation of vortex-ring discrete solitons in 2D photonic lattices. Phys. Rev. Lett. 92, 123904 (2004).

[29] N.K. Efremidis, J. Hudock, D.N. Christodoulides, J.W. Fleischer, O. Cohen, and M. Segev, "Two-Dimensional Optical Lattice Solitons", Phys. Rev. Lett. 91, 213906 (2003).

[30] E.A. Ostrovskaya and Y.S. Kivshar, "Photonic crystals for matter waves: BoseEinstein condensates in optical lattices", Opt. Exp. 12, 19-29 (2004).

[31] D.J.B. Lloyd and A.R. Champneys, "Efficient numerical continuation and stability analysis of spatiotemporal quadratic optical solitons," SIAM J. Sci. Comput. 27, 759-773 (2005).

[32] J.J. Garcia-Ripoll, V.M. Perez-Garcia, E.A. Ostrovskaya, and Y.S. Kivshar, "Dipolemode vector solitons", Phys. Rev. Lett. 85, 82 (2000).

[33] J. Yang and D.E. Pelinovsky, "Stable vortex and dipole vector solitons in a saturable nonlinear medium." Phys. Rev. E 67, 016608 (2003). 
(a)

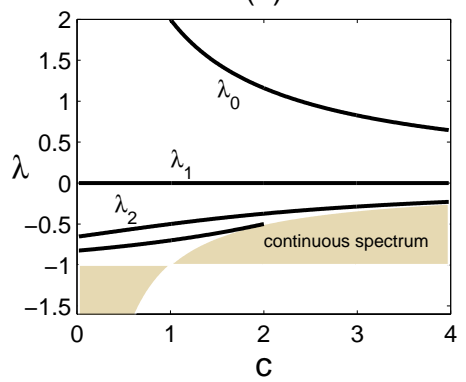

(c)

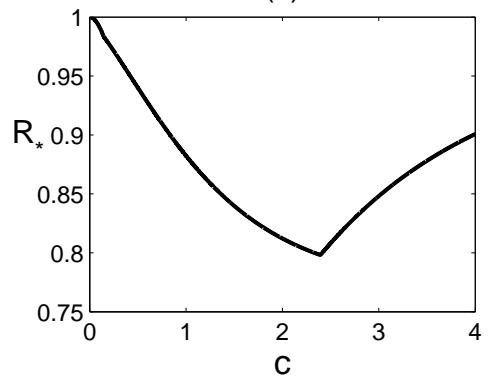

(b)

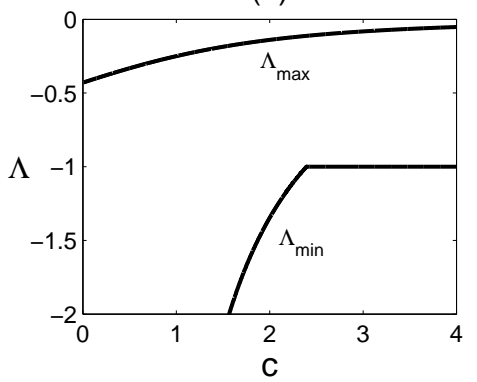

(d)

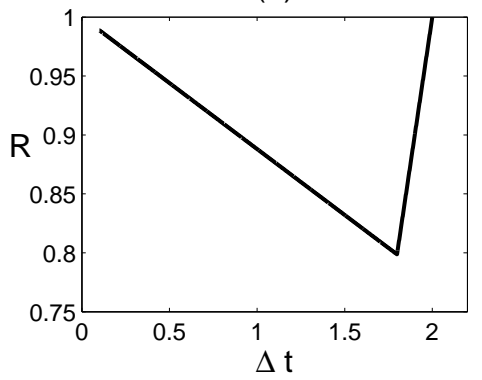

Figure 1: Analysis of the SOM (4) applied to the soliton (17) of the NLS equation (16). (a) Eigenvalues of operator $M^{-1} L_{1}$, with $M$ given in (18), versus the acceleration parameter $c$; (b) maximum and minimum (nonzero) eigenvalues of the iteration operator $\mathcal{L}=-\left(M^{-1} L_{1}\right)^{2} ;$ (c) graph of convergence factor $R_{*}(c)$ versus $c$; its minimum occurs at $c=c_{\text {opt }}=6-\sqrt{13} ;$ (d) convergence factor function $R(\Delta t ; c)$ versus $\Delta t$ at $c=c_{\text {opt }}$. 
(a)

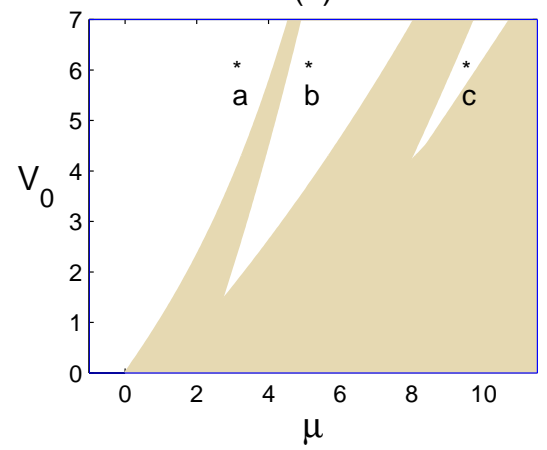

(c)

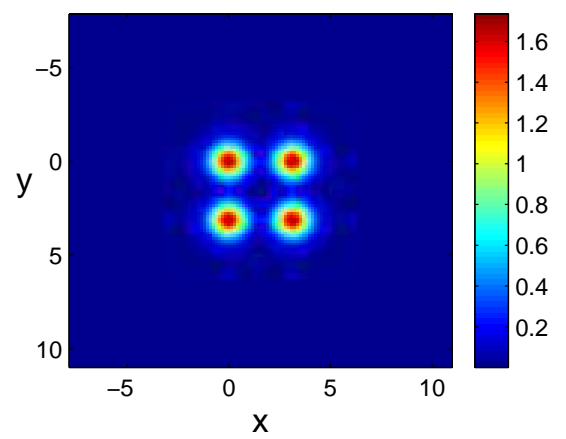

(b)

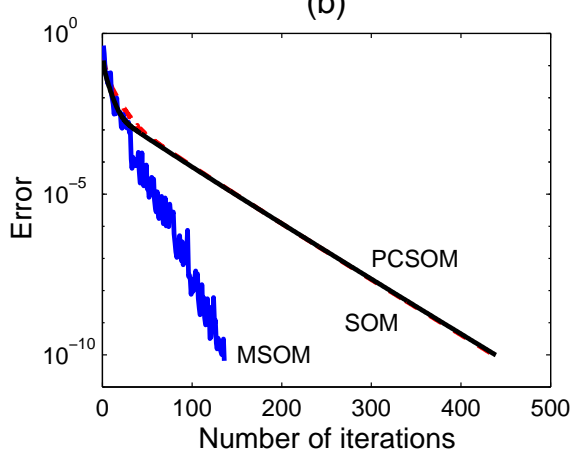

(d)

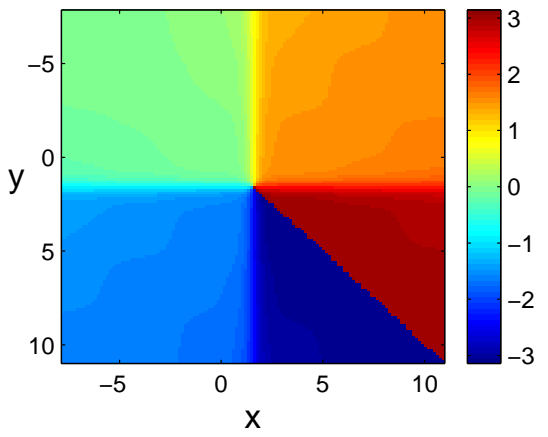

Figure 2: (a) Bandgap structure of Eq. (1); (b) error diagrams for SOM (4), MSOM (1), (5) and PCSOM (3)-(5) at optimal $c$ and $\Delta t$ values (see text); SOM and PCSOM are almost indistinguishable; (c, d) a vortex soliton in the semi-infinite bandgap of Eq. (1) with focusing nonlinearity. Here $\mu=3(P=14.6004)$, marked by letter 'a' in panel (a). (c) is the amplitude $(|U|)$ distribution, and (d) the phase distribution. 
(a)

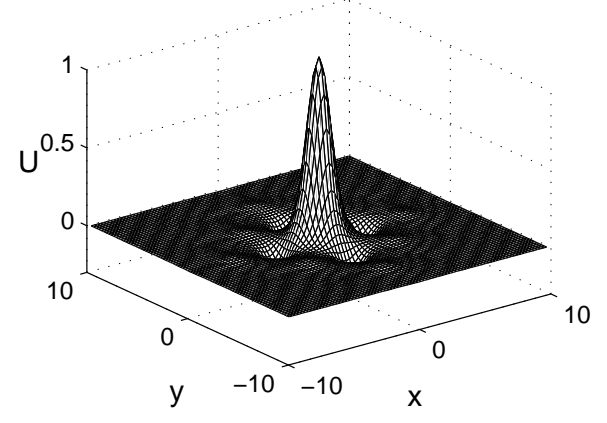

(b)

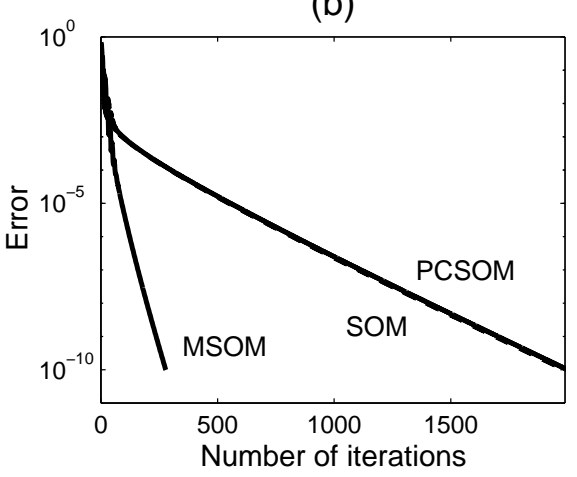

Figure 3: (a) A solitary wave in the first bandgap of Eq. (1) with defocusing nonlinearity at $\mu=5(P=2.4936)$, marked by letter 'b' in Fig. 2(a); (b) error diagrams for SOM (4), MSOM (1), (5) and PCSOM (3)-(5) at optimal $c$ and $\Delta t$ values (see text); SOM and PCSOM are almost indistinguishable. 
(a)

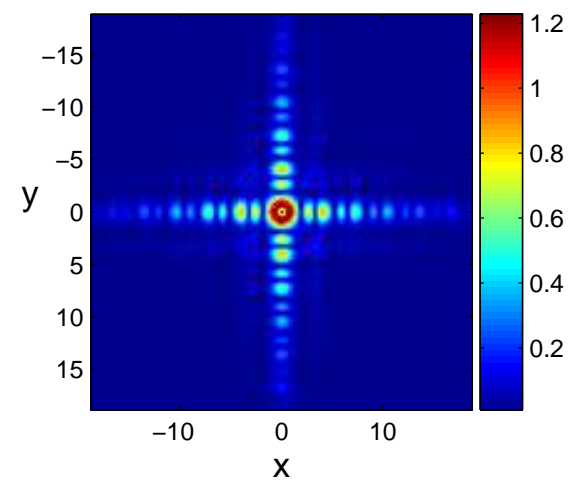

(b)

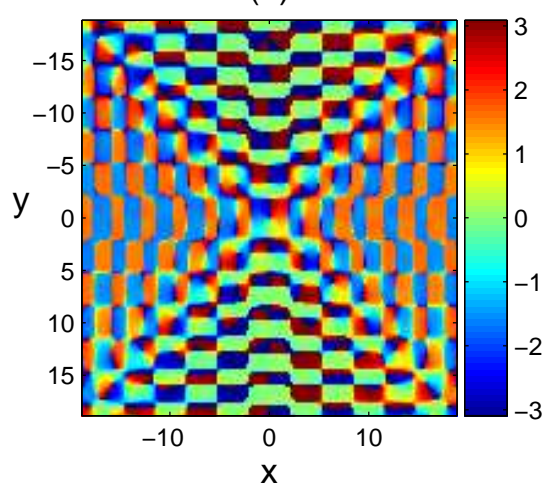

(c)

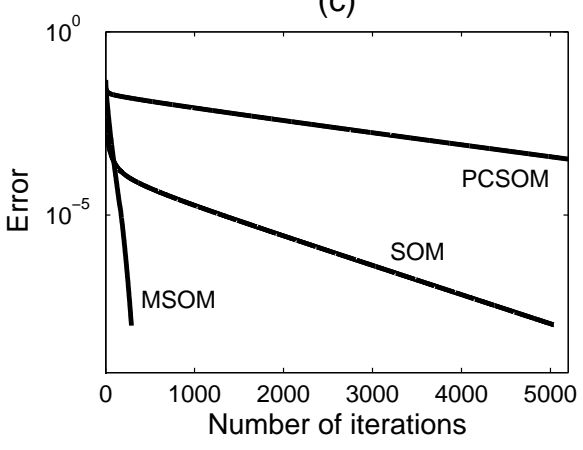

Figure 4: (a, b) A vortex-soliton solution in the second bandgap of Eq. (1) with defocusing nonlinearity at $\mu=9.4(P=18.3578)$, marked by letter 'c' in Fig. 2(a). (a): amplitude distribution; (b) phase distribution. (c) error diagrams for SOM (4), MSOM (1), (5) and PCSOM (3)-(5) at optimal $c$ and $\Delta t$ values (see text). 
(a)

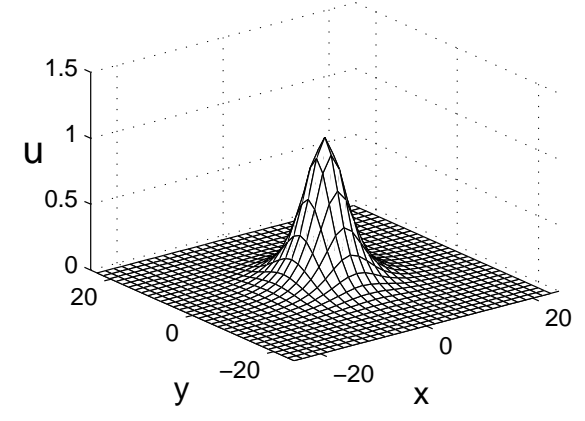

(b)

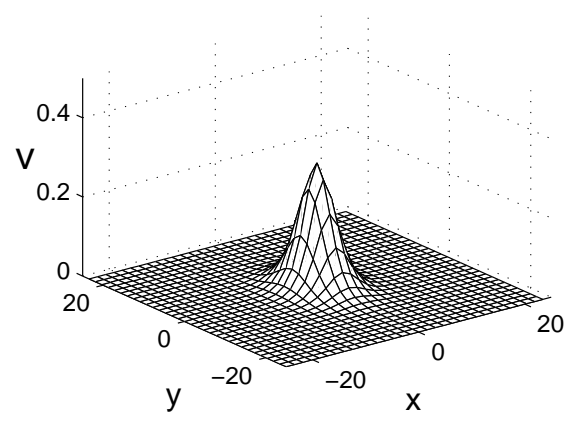

(c)

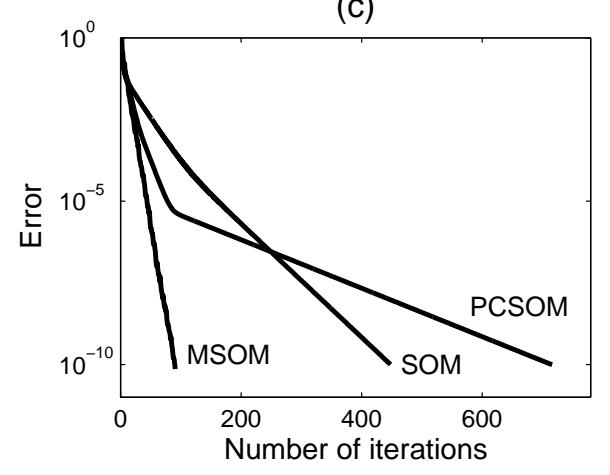

Figure 5: (a, b) A fundamental solitary wave in the SHG model (9)-(10) at $\mu=0.1$ $(P=47.3744)$; (c) error diagrams for SOM (4), MSOM (1), (5) and PCSOM (3)-(5) at optimal scheme parameters (see text). 
(a)

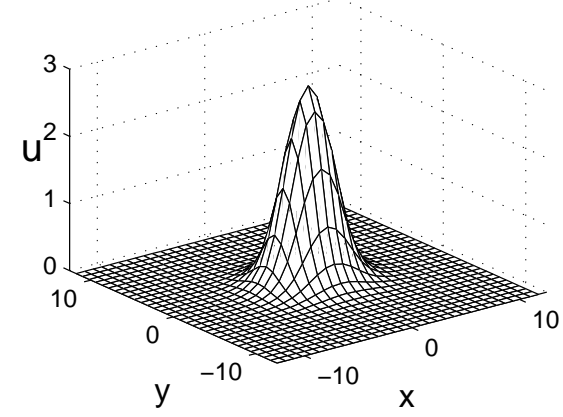

(b)

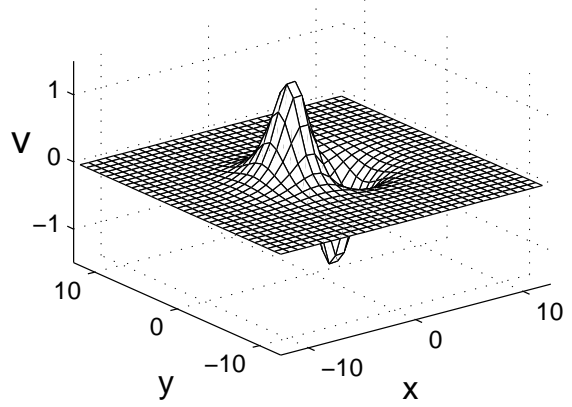

(c)

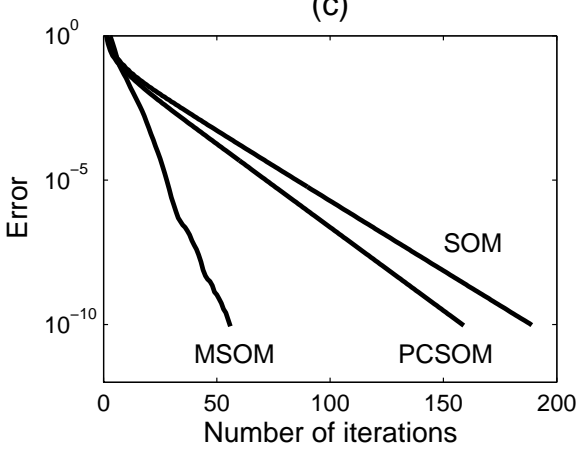

Figure 6: (a, b) A dipole-mode vector solitary wave in the coupled NLS system (13)-(14) at $\mu_{1}=1$ and $\mu_{2}=0.5\left(P_{1}=85.3884, P_{2}=29.1751\right)$; (c) error diagrams for SOM (4), MSOM (1), (5) and PCSOM (19)-(21) at optimal scheme parameters (see text). 
(a)

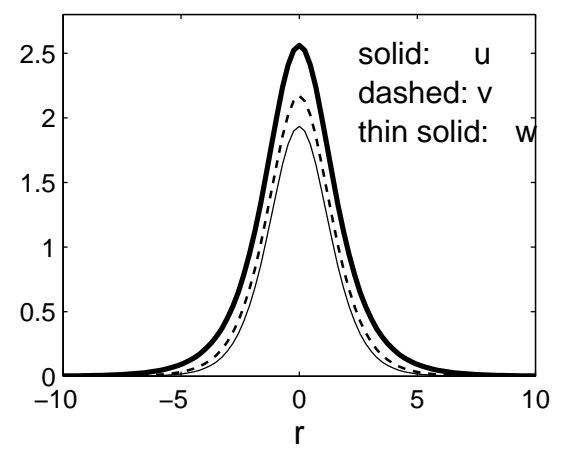

(b)

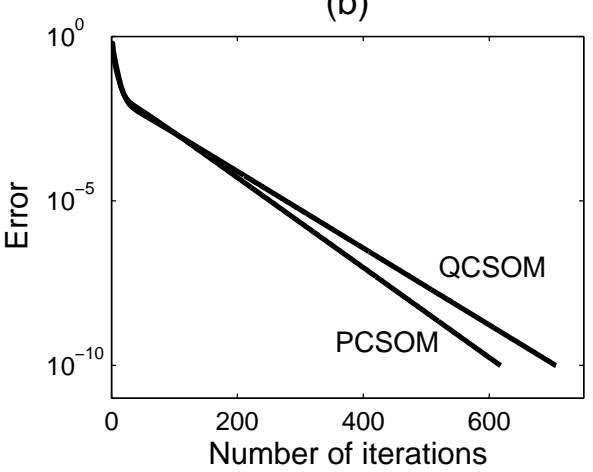

Figure 7: (a, b) A fundamental soliton in the three-wave model (17)-(19) at $\mu_{1}=0.5$ and $\mu_{2}=1$, where $Q_{1}=\langle u, u\rangle+\langle w, w\rangle=66.3096$, and $Q_{2}=\langle v, v\rangle+\langle w, w\rangle=47.2667$; (c) error diagrams for PCSOM (34)-(38) and QCSOM (49) at optimal scheme parameters (see text).

(a)

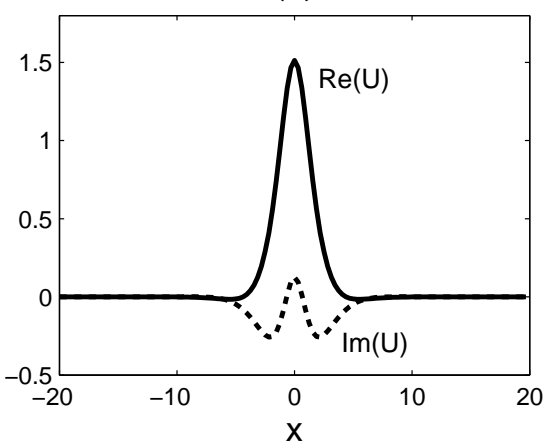

(b)

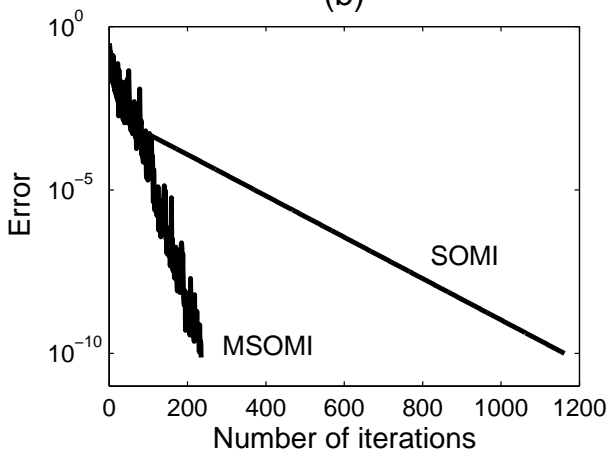

Figure 8: (a) An isolated solitary wave in the Ginzburg-Landau equation (25) with $\gamma_{0}=0.3$ and $\gamma_{1}=1$; (b) error diagrams of SOMI (1)-(2) and MSOMI (6)-(10) at optimal $c$ and $\Delta t$ values (see text). 